\title{
Dynamics of the large-scale structures and associated noise emission in airfoil slats
}

\author{
Daniel S. Souza ${ }^{1,3,4, \dagger}$, Daniel Rodríguez ${ }^{2,3,5, \dagger, ~ F e r n a n d o ~ H . ~ T . ~ H i m e n o ~}{ }^{3}$ \\ and Marcello A. F. Medeiros ${ }^{3}$
}

${ }^{1}$ Department of Thermal and Fluid Sciences, Federal University of São João del Rei, Praça Frei Orlando, 170, São João del Rei, Brazil

${ }^{2}$ ETSIAE-UPM (School of Aeronautics), Universidad Politécnica de Madrid, Plaza del Cardenal Cisneros 3, 28040 Madrid, Spain

${ }^{3}$ Department of Aeronautical Engineering, University of São Paulo, Av. Trabalhador São Carlense, 400, São Carlos, SP 13566-590, Brazil

${ }^{4}$ UNESP - São Paulo State University, Campus São João da Boa Vista, São João da Boa Vista, SP 13876-750, Brazil

${ }^{5}$ Mechanical Engineering Department, Universidade Federal Fluminense (UFF), Niteroi, RJ 24210-240, Brazil

(Received 6 May 2018; revised 14 June 2019; accepted 16 June 2019;

first published online 26 July 2019)

We investigate the slat narrowband peak noise generating mechanism. Unsteady flow data were generated by a lattice-Boltzmann-based commercial code for four configurations, accounting for variations of the airfoil angle of attack and slat overlap. Comparison with experimental results indicates that the aspects of the flow field relevant for the generation of the narrowband peaks were accurately captured. Frequency-domain proper orthogonal decomposition (POD) is applied to identify dominant large-scale structures in the frequency range dominated by the peaks. The combined use of the two POD metrics, namely, the turbulent kinetic energy in the turbulent flow region and the acoustic pressure in the far field, demonstrated that the structures most correlated with the noise resemble spanwise coherent Kelvin-Helmholtz vortices which dominate the slat cove only at the frequency of the narrowband peaks. Time evolution of the structures educed using the acoustic pressure correlation provides detailed evidence of the hydrodynamic and acoustic steps of a Rossiter-like feedback mechanism between the slat cusp and trailing edge. The combined analysis of results for the different slat configurations provides an explanation for the effect of the slat configuration on the amplitude of the narrowband peaks observed in previous studies, particularly the influence of the main-element suction peak.

Key words: aeroacoustics, hydrodynamic noise, low-dimensional models

\section{Introduction}

Along with landing gears and flap side edge, the slat is one of the most important airframe noise sources for commercial airplanes (Fink 1979; Crighton 1991). During

$\dagger$ Email address for correspondence: daniel.s.souza@unesp.br

$\ddagger$ Present address: ETSIAE-UPM, Universidad Politécnica de Madrid, Spain 
the landing phase, the slat-generated noise can be comparable to the engine noise (Dobrzynski 2010). Therefore, understanding the mechanisms associated with the generation of slat noise is of fundamental importance for the design of new quieter commercial airplanes that fulfil the increasingly stringent noise regulations.

Three basic components have been identified in the spectrum of slat noise in wind tunnel experiments using reduced-scale models, namely a hump at high frequency, a broadband portion ranging from relatively low-to-mid frequencies and a series of narrowband peaks in the frequency range of maximum broadband noise (Imamura et al. 2009). Typical ranges of Strouhal number $S t$, based on the slat chord $c_{\text {slat }}$ and free-stream velocity $U_{\infty}$, are given as follows: the high-frequency hump often occurs at $S t \approx 20$; the broadband portion ranges from $S t \approx 0.1$ up to $S t \approx 15$, where the spectrum reaches a local minimum, while its maximum is typically observed in the same range where the narrowband peaks appear, between $S t=1$ and 8 .

Complementary wind tunnel experiments and Navier-Stokes simulations allowed the identification of slat trailing-edge vortex shedding as the mechanism responsible for the high-frequency hump (Khorrami, Berkman \& Choudhari 2000; Choudhari et al. 2002). However, it is now generally accepted that the high-frequency hump is an artefact of the relatively thick trailing edge of reduced-scale models, owing to machining precision limitations, and does not scale to real airplanes (Dobrzynski \& Pott-Pollenske 2001). On the other hand, the broadband noise has been associated with the growth of disturbances in the mixing layer that separates the accelerated gap flow from the slat cove recirculation zone (Dobrzynski \& Pott-Pollenske 2001; Khorrami, Singer \& Berkman 2001).

It has been argued that the series of narrowband peaks is also a model effect due to the low Reynolds number in wind tunnel experiments (Dobrzynski 2010). This is supported by the Dobrzynski et al. (1998) measurements, which achieved an almost complete suppression of the peaks by forcing the transition of the boundary layer slightly upstream of the slat cusp. However, another attempt at reducing the peaks by tripping the slat pressure side boundary layer by Murayama et al. (2014) did not succeed. Moreover, wind tunnel measurements by Herr et al. (2015) with Reynolds numbers up to 5 million, and Navier-Stokes simulations by Lockard \& Choudhari (2012) at real-scale Reynolds numbers show similar series of narrowband low-frequency peaks. Therefore, the relevance of the narrowband peaks to full-scale slat noise remains an open question. The present paper is devoted to this slat noise component.

The effect of the airfoil's angle of attack on the low-mid-frequency slat noise has been thoroughly investigated (Kolb et al. 2007; Imamura et al. 2009; Murayama et al. 2014; Pagani, Souza \& Medeiros 2016; Amaral et al. 2017). The consistent observation in this regard is that the noise at this range decreases significantly and the frequencies of the narrowband peaks decrease slightly as the angle of attack increases. Wind tunnel experiments by Pascioni \& Cattafesta (2018) and simulations by Pagani et al. (2016) indicate that, as the angle of attack increases, the spanwise coherence of the mixing-layer disturbances at the peak frequencies decreases significantly.

Concerning the influence of different slat settings, Mendoza, Brooks \& Humphreys (2002) show a reduction of the slat noise in the low-mid-frequency range as the slat approximates the main element, i.e. simultaneously reducing the gap and increasing the overlap. Measurements by Pott-Pollenske, Alvarez-Gonzalez \& Dobrzynski (2003) and computations by Ewert et al. (2010) also indicate that the low-mid-frequency noise reduces as the gap is reduced. Pott-Pollenske et al. (2003) attributed this reduction to flow speed drop in the slat gap region. Pagani, Souza \& Medeiros (2017) 
presented a comprehensive study of the effect of slat gap, overlap and deflection angle and indicated that the amplitude of the narrowband peaks is, in general, inversely related to the magnitude of the main-element suction peak.

The relation between coherent vortical structures developing in the cove turbulent field and low-frequency noise has been discussed by a number of authors. Khorrami, Choudhari \& Jenkins (2004) identified the intermittent ejection of the mixing-layer spanwise vortices through the slat gap as the origin of acoustic waves of low frequencies. Dobrzynski \& Pott-Pollenske (2001) and Imamura et al. (2008) also associated the emission at low frequency with unsteadiness in the slat gap region related to the mixing-layer disturbances. Dobrzynski \& Pott-Pollenske (2001) showed good scalability of the low-frequency slat noise acoustic power in wind tunnel measurements with $U_{\infty}^{5}$ and suggested that it is generated by the interaction of the slat cove vortices with the slat trailing edge (Ffowcs-Williams \& Hall 1970). This observation led them to relate the slat noise emission to a dipole aligned to the trailing edge. Power laws close to 5 have also been observed by other researchers (Pérennè \& Roger 1998; Imamura et al. 2009; Murayama et al. 2014; Pagani et al. 2016). The double lobe directivity pattern recovered in the simulations by Lockard \& Choudhari (2009) and Ewert et al. (2010) further supports Dobrzynski and Pott-Pollenske's argument.

Hein et al. (2007) investigated if pure acoustic resonances could be the origin of the low-frequency narrowband peaks. However, the idea of acoustic resonances contrasts with the observation that peak frequencies scale fairly well with the Strouhal number, which is based on the flow velocity and not on the speed of sound. More commonly, the selection of the low-frequency narrowband peaks is attributed to a mechanism similar to cavity modes (Rossiter 1966), involving vortices generated in the slat cove mixing layer, their impingement or passage close to an edge and an acoustic feedback. Different semi-empirical models for their prediction have been proposed based on this idea. Kolb et al. (2007) applied the semi-empirical equation by Heller \& Bliss (1975), a modified version of the one by Rossiter (1966), to predict the frequency of peaks and attained a reasonable agreement with wind tunnel experiments for one airfoil angle of attack. However, this equation fails to capture the effect of angle of attack which the authors attributed to the lack of detailed information of the slat cove flow field. Deck \& Laraufie (2013) used the predictions of the peak frequencies given by different equations, showing good agreement with the frequencies identified in the cove flow computed with simulations for $4^{\circ}$ angle of attack. Terracol, Manoha \& Lemoine (2016) derived an equation tailored for the slat geometry to predict the peak frequencies, which will be discussed below. Their results present good agreement with time-accurate simulation data also for an angle of attack of $4^{\circ}$.

Frequency-domain two-dimensional proper orthogonal decomposition (POD) using data from a three-dimensional simulation carried out by Souza et al. (2015) shows that flow structures resembling Kelvin-Helmholtz $(\mathrm{K}-\mathrm{H})$ vortices are the most correlated with the pressure fluctuations at a line just outside the slat cove, at the frequency of a narrowband peak. Richard, Wilkins \& Hall (2018) performed time-domain POD computations based on sectional particle image velocimetry (PIV) data of the flow in the slat cove, and educed mixing-layer vortices at a frequency that is consistent with Rossiter-like modes predicted from Terracol's model. PIV measurements by Pascioni \& Cattafesta (2018) also revealed coherent mixing-layer fluctuations associated with the narrowband peaks.

Several numerical methodologies have been employed to simulate the flow over high-lift airfoils with focus on slat noise. Methods based on the reconstruction of the 
noise source from turbulence information given by steady Reynolds-averaged NavierStokes computations have been used (Ewert 2008; Dierke et al. 2011). However, typically unsteady zonal approaches are used, either unsteady Reynolds-averaged Navier-Stokes with the production of modelled turbulent kinetic energy turned off in the slat cove region (Khorrami, Singer \& Lockard 2002; Lockard \& Choudhari 2009), or hybrid Reynolds-averaged Navier-Stokes/large eddy simulation (Deck 2005; Imamura et al. 2008; Deck \& Laraufie 2013). In particular, the experiences by Jenkins, Khorrami \& Choudhari (2004), Khorrami et al. (2004) and Choudhari \& Khorrami (2007) showed that the turbulent spanwise structure must be taken into account in order to properly reproduce the slat noise emission. Simulations based on the lattice-Boltzmann method (LBM) have demonstrated success comparable to the unsteady Navier-Stokes-based methods (Satti et al. 2008; Aflalo et al. 2010; Pagani et al. 2016).

The present investigation presents unsteady simulations of the flow and emitted noise of typical high-lift configurations. The main objectives are (i) to identify the large-scale structures most correlated to the slat noise at the frequency range of the narrowband peaks; (ii) to understand the effects of geometric parameters on the dynamics of these structures; (iii) to analyse in more detail the feedback mechanism associated with the narrowband peaks; and (iv) to explain the effect of the geometric parameters on the noise emission. A commercial code based on the lattice-Boltzmann method is used to perform the simulations. Four different geometric configurations are considered, including different slat settings and airfoil angle of attack. The cases are selected from the experiments of Pagani et al. (2017) with the aim of covering different slat noise regimes. Proper orthogonal decomposition in the frequency domain (sometimes referred to as spectral POD) is applied in the analysis of the simulation data. Two different correlation metrics are used, unveiling different aspects of the flow physics. The first metric is the usual turbulent kinetic energy norm, that recovers the most energetic flow structures. The second metric considers the acoustic fluctuations in a plane located below the airfoil, in the acoustic far field, and recovers the turbulent flow structures more correlated with the radiated noise.

The paper is organized as follows: in $\$ 2$, the flow simulation methodology is described and validated. In $\S 3.1$, we describe the POD method with the particularities of the present implementation, while in $\S 3.2$ we discuss the rank and shape of the structures educed by the POD computations. The evidences of a feedback mechanism in the flow simulation and particularly in the educed large-scale structures are discussed in $\S 4.1$ and an explanation for the effect of the slat geometrical parameters on the narrowband peak amplitudes is proposed in $\S 4.2$. The main conclusions are summarized in $\S 5$.

\section{Unsteady simulations}

\subsection{Lattice-Boltzmann method and PowerFLOW code}

The lattice-Boltzmann-based commercial code PowerFLOW 5.0a is used to simulate the flow around the high-lift configuration and to resolve the aeroacoustic noise sources in the flow around the slat. PowerFLOW solves the discrete Boltzmann equation with the Bhatnagar-Gross-Krook approximation for the collision term (Bhatnagar, Gross \& Krook 1954),

$$
f_{k}\left(\boldsymbol{x}+\boldsymbol{\xi}_{k} \Delta t, \boldsymbol{\xi}_{k}, t+\Delta t\right)-f_{k}\left(\boldsymbol{x}, \boldsymbol{\xi}_{k}, t\right)=-\frac{\Delta t}{\tau}\left[f_{k}\left(\boldsymbol{x}, \boldsymbol{\xi}_{k}, t\right)-f_{k}^{e q}\left(\boldsymbol{x}, \boldsymbol{\xi}_{k}, t\right)\right]
$$


which is first-order accurate in time (He \& Luo 1997). The distribution function, $f_{k}$, represents the probability density of finding a particle in $\boldsymbol{x}$, with velocity $\boldsymbol{\xi}_{k}$ at instant $t$. The relaxation time of the collision term approximation is denoted by $\tau$ and $\Delta t$ is the time step. The equilibrium distribution, $f_{k}^{e q}$, corresponding to the $k$ th discrete particle velocity vector $\boldsymbol{\xi}_{k}$, is defined as

$$
f_{k}^{e q}=\rho W_{k}\left[1+\frac{\left(\boldsymbol{\xi}_{k} \cdot \boldsymbol{V}\right)}{T}+\frac{\left(\boldsymbol{\xi}_{k} \cdot \boldsymbol{V}\right)^{2}}{2 T^{2}}-\frac{\boldsymbol{V}^{2}}{2 T}+\frac{\left(\boldsymbol{\xi}_{k} \cdot \boldsymbol{V}\right)^{3}}{6 T^{3}}-\frac{\left(\boldsymbol{\xi}_{k} \cdot \boldsymbol{V}\right)}{2 T^{2}} \boldsymbol{V}^{2}\right],
$$

where $T$ is the lattice temperature, which is a constant equal to $1 / 3$, and $W_{k}$ are weighting factors. A set of 19 symmetrically oriented velocity vectors are used for the discretization of the velocity space. The magnitude of each of the velocity vectors is equal to the distance between the centre of the neighbouring cells divided by the time step, and the weight factors are 1/18 for the vectors in the coordinate directions, $1 / 36$ for the bi-diagonal velocity vectors and 1/3 for the null vector (Li et al. 2004). The density $\rho$ and macroscopic velocity $\boldsymbol{V}$ are retrieved by the zeroth- and first-order moments, respectively,

$$
\rho(\boldsymbol{x}, t)=\sum_{k} f_{k}\left(\boldsymbol{x}, \boldsymbol{\xi}_{k}, t\right)
$$

and

$$
\rho(\boldsymbol{x}, t) \boldsymbol{V}(\boldsymbol{x}, t)=\sum_{k} \boldsymbol{\xi}_{k} f_{k}\left(\boldsymbol{x}, \boldsymbol{\xi}_{k}, t\right) .
$$

With the equilibrium distribution given by (2.2), the Chapman-Enskog expansion leads to the compressible Navier-Stokes equations for low Mach numbers together with the relation $p=\rho T$ (Wolf-Gladrow 2000).

To model the small-scale turbulent dissipation, PowerFLOW uses a modified version of the renormalization group (RNG) $k-\epsilon$ turbulence model that allows the lattice-Boltzmann method to resolve the large-scale structures in regions of high vorticity (Fares 2006). The code also features a proprietary wall model to determine the velocity field in the cells next to solid walls in turbulent flows, which dynamically accounts for pressure gradients (Teixeira 1998).

The acoustic field associated with the simulated flow is computed by means of an algorithm based on the Farassat's 1A formulation (Farassat \& Succi 1980) for the Ffowcs Williams-Hawkings (FW-H) equation, adapted through a Galilean transformation to consider a wind tunnel flow configuration (Brès, Pérot \& Freed 2010; Najafi-Yazdi, Brès \& Mongeau 2011).

\subsection{Description of simulation model}

The simulations consider high-lift configurations based on the three-element MD30P30N airfoil. This is an optimized configuration in a typical landing arrangement, developed by McDonnell Douglas in the early 1990s. It was primarily used in wind tunnel experiments to characterize the flow over high-lift configurations at realistic flight conditions and to provide a database for the validation of computational fluid dynamics codes (Valarezo et al. 1991; Chin et al. 1993; Klausmayer \& Lin 1994; Spaid \& Lynch 1996). Since the early 2000s, this geometry has been extensively used in the study of the aeroacoustic characteristics of high-lift airfoils (Jenkins et al. 2004; Khorrami et al. 2004; Choudhari \& Khorrami 2007; Lockard \& Choudhari 2009; Murayama et al. 2014; Pagani et al. 2016, 2017; Zhang et al. 2017; Pascioni \& Cattafesta 2018). 


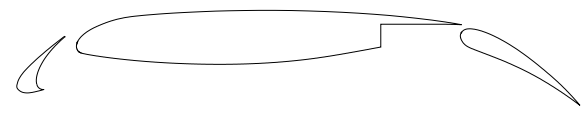

FIGURE 1. Cross-section of the MD30P30N high-lift configuration.

$\begin{array}{lcccc} & \text { Gap (\%) } & \text { Overlap (\%) } & \text { Deflection (deg.) } & \text { Chord (\%) } \\ \text { Slat } & 2.95 & -2.50 & 30 & 15 \\ \text { Flap } & 1.25 & 0.25 & 30 & 30\end{array}$

TABLE 1. Geometrical settings of the MD30P30N airfoil used in this investigation. Percentages are relative to the stowed chord $\left(c_{s t}\right)$.

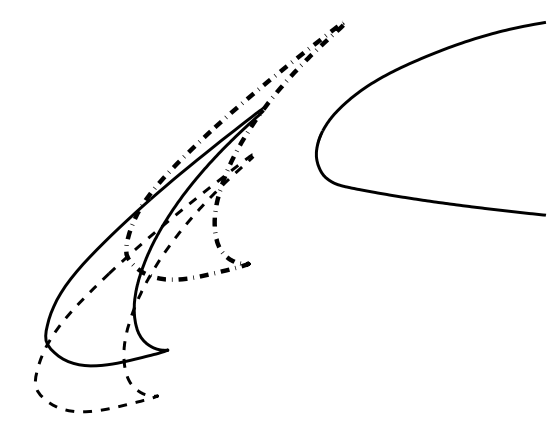

FIGURE 2. Schematic view of the simulated slat settings. Solid line: olA, dashed line: olB and dotted line: olC.

Figure 1 shows the cross-section of the MD30P30N configuration. The geometry simulated has sharp trailing edges for the main element and flap. The slat trailing edge is truncated with a final thickness equal to $0.092 \%$ of the airfoil stowed chord $\left(c_{s t}\right)$. Table 1 gives the values of slat and flap setting parameters for the MD30P30N airfoil. The slat and flap chords, as well as the gap and overlap distances are given as a percentage of the airfoil stowed chord.

Besides the original, baseline MD30P30N configuration, two other slat arrangements are considered here, with overlap $o_{s}$ equal to $-2.95 \%$ and $1 \%$ of the stowed chord. For the baseline configuration, two angles of attack $\alpha$ are considered, namely $4^{\circ}$ and $8^{\circ}$. Hereinafter, these cases are referred to as olA $\alpha 4$ and olA $\alpha 8$, respectively. The configurations with modified slat overlap are simulated only at $\alpha=4^{\circ}$. The one with overlap equal to $-2.95 \%$ of the stowed chord is referred to as olB $\alpha$, and the configuration with overlap equal to $1.00 \%$ of $c_{s t}$, as olC $\alpha 4$. Figure 2 shows schematically the three slat settings considered in the simulations.

The flow conditions and computational domain of the simulations are set in order to reproduce the wind tunnel experiments of Pagani et al. (2016). A schematic cross-sectional view of the three-dimensional computational domain is shown in figure 3. The vertical dimension is chosen to match the corresponding length in the reference wind tunnel experiments, $1.7 \mathrm{~m}$. The extension of the domain in the streamwise direction (coordinate $x$ ) is chosen following the domain sensitivity study described in $\S 2.3$. The inflow speed and stowed chord in all simulations respectively match the maximum inflow speed used in the wind tunnel experiments, $34 \mathrm{~m} \mathrm{~s}^{-1}$, 


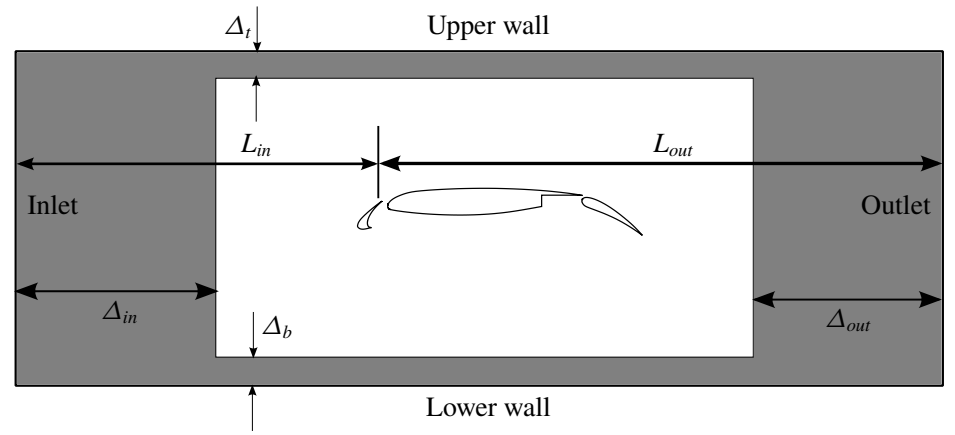

FIGURE 3. Schematic cross-section representation of the computational domain used in the lattice-Boltzmann method (LBM) simulations. In grey, region of high non-physical viscosity (anechoic layer). Note the actual proportions in figure 4.

and the model stowed chord, $c_{s t}=0.5 \mathrm{~m}$. The Reynolds number based on the stowed chord was 1 million and the Mach number was $M a=0.1$, also corresponding to the experiments with the maximum inflow speed.

Uniform velocity is prescribed as the inflow boundary condition. To provide the necessary inflow boundary condition to the turbulence model, the turbulent intensity (defined by the ratio between root mean square of the turbulent velocity fluctuations and the mean inflow velocity) and turbulent length scales are set to $9 \times 10^{-4}$ and $1 \mathrm{~mm}$ respectively for all cases. These values are the same employed for simulations carried out by Simões, Souza \& Medeiros (2011) to reproduce the conditions (wind tunnel dimensions, Reynolds and Mach numbers) of the NASA Basic Aerodynamics Research Tunnel (BART) (Jenkins et al. 2004). The imposed turbulence intensity does not match the corresponding value of the experiments by Pagani et al. (2016) and Pagani et al. (2017), which was approximately $0.2 \%$ (Dantas et al. 2010). However, this difference is not expected to impact the results significantly given the relative lack of sensitivity of the dominant slat noise to the turbulence level of the slat boundary layer demonstrated by wind tunnel experiments (Murayama et al. 2014) and LBM computations (Simões et al. 2011). The upper and lower domain boundaries are modelled as free-slip walls and the airfoil surfaces are modelled as no-slip walls. At the outflow, uniform pressure equal to 1 atm is prescribed, while the velocity direction is forced to be the same as at the inlet. Periodic conditions are applied in the spanwise direction, which approximate a wing of infinite span if the spanwise domain is long enough (see $\$ 2.3$ ).

A region of artificially high viscosity is set in the outer portion of the computational domain by adjusting the relaxation time in that region (represented by the grey zone in figure 3) to prevent acoustic wave reflections. Note that the reduced reflections at the top and bottom boundaries do not represent the reference experiments, since the corresponding tunnel walls were rigid. However, without the anechoic layer, the time to dissipate the acoustic waves generated by the strong initial transient caused by the sudden appearance of an airfoil in an otherwise uniform flow field would be prohibitive. There is some concern that acoustic experiments in closed section wind tunnels may lead to wave reflections that can affect noise measured by a microphone. This effect was circumvented in the reference experiments with the use of beamforming which can remove the image sources from the region of integration of the sources (Amaral et al. 2019). In the simulations, the sound is obtained only 
(a)

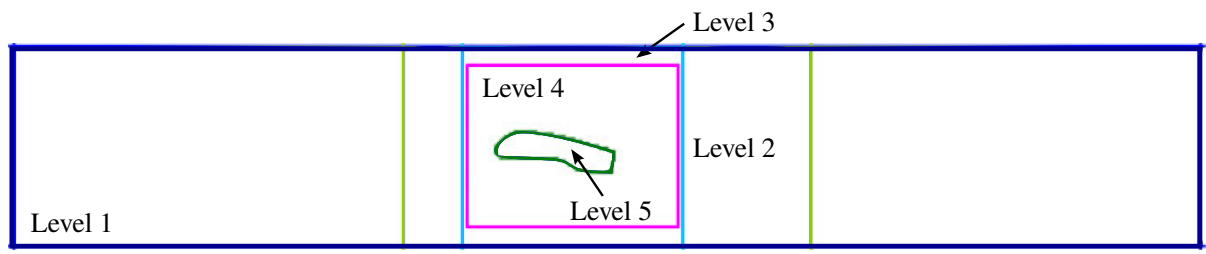

(b)

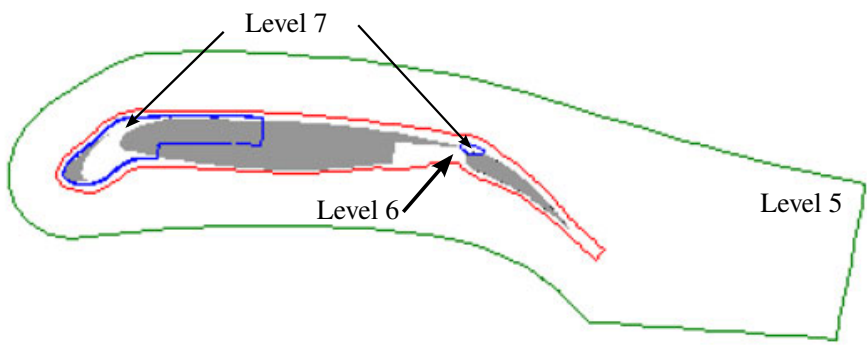

FIGURE 4. (Colour online) Regions of different grid refinement levels. (a) Zoomed out view showing regions of lower refinement levels and $(b)$ detail of the airfoil region.

from sources on the model surface, which also does not take into account the images that could be produced by the boundaries of the computational domain.

The initial condition at the physical viscosity region, is set to be the inlet velocity plus a randomly distributed velocity field whose magnitude is limited to $20 \%$ of the inlet velocity. The addition of the random component triggers the inherent flow instabilities and reduces the time to reach a fully developed turbulent flow. At the anechoic layer, the initial velocity is equal to the inlet velocity. The computational domain is segmented in seven regions with different levels of grid refinement. Figure 4 shows schematically the boundaries of these regions. This grid topology was also chosen based on the computations performed by Simões et al. (2011) at the BART conditions and resulted in good comparison with NASA's experiments (Souza et al. 2015).

\subsection{Sensitivity of results to computational parameters}

Table 2 summarizes the computational parameters used in the different simulations presented in this paper. The dependence of the numerical results on the computational parameters is investigated considering primarily the olA $\alpha 4$ configuration.

To verify that the results are independent of the computational domain's streamwise extension and anechoic layer's thickness, the power spectral densities (PSDs) of the pressure at the reattachment point and a point on the slat upper surface are compared in figure 5 for cases olA $\alpha 4-\operatorname{dom} X 1, o l A \alpha 4-\operatorname{dom} X 2, o l A \alpha 4-\operatorname{dom} X 3$ and $o l A \alpha 4-\operatorname{dom} Y 2$. The upper surface point is chosen, based on previous studies by Lockard \& Choudhari (2009) and Simões et al. (2011) showing that the unsteady pressure field at this position is highly influenced by acoustic waves emanating from the slat and is, therefore, very similar in shape to the far-field spectrum. Statistical convergence study based on case olA $\alpha 4-\operatorname{dom} X 3$, which has total simulation time of approximately 263 slat through times (STT), showed that the initial transient lasted 36 STT and statistically independent spectra are computed using a 95 STT long interval. 

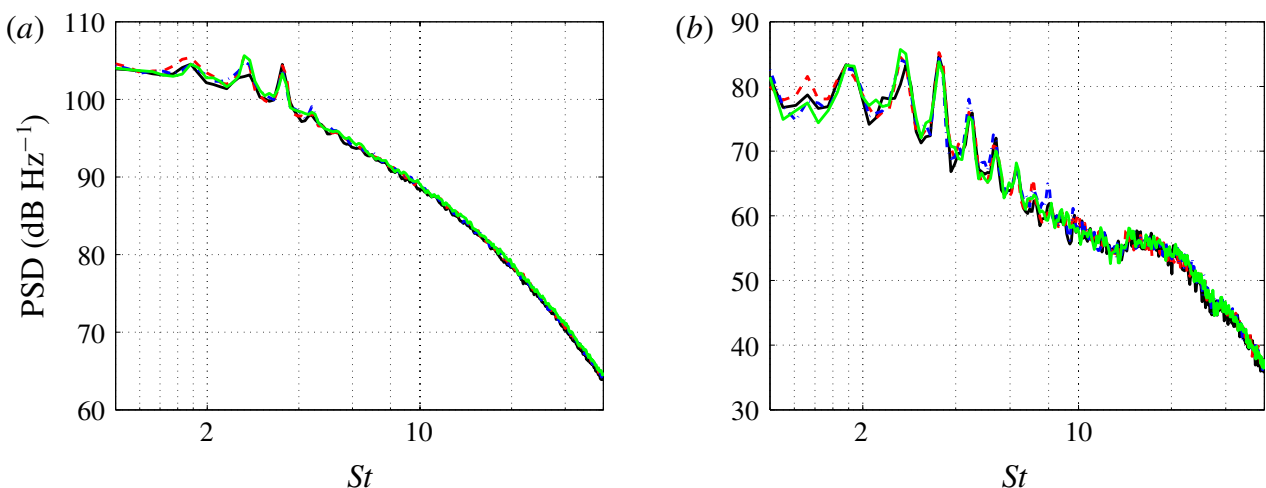

FiguRE 5. (Colour online) Power spectral density (PSD) of pressure fluctuations at $(a)$ the reattachment point and $(b)$ a point on the slat upper surface, for different domain extents in the streamwise direction and different thicknesses of the anechoic layer. Solid black line: olA $\alpha 4-\operatorname{dom} X 1$, dashed red line: olA $\alpha 4-\operatorname{dom} X 2$, dotted blue line: olA $\alpha 4-\operatorname{dom} X 3$ and solid green line: $o l A \alpha 4-\operatorname{dom} Y 2$.

$\begin{array}{lcccccccccc}\text { Case } & L_{\text {in }} & L_{\text {out }} & \Delta_{\text {in }} & \Delta_{\text {out }} & \Delta_{t / b} & L_{z} & \Delta x_{\min } & \Delta t_{\min }(\mathrm{s}) & o_{s} & \alpha \text { (deg.) } \\ \text { olA } \alpha 4 \text {-dom } X 1 & 10 & 14 & 5.21 & 7.25 & 0.18 & 0.102 & 4 \times 10^{-4} & 3.3 \times 10^{-7} & -0.025 & 4 \\ \text { olA } \alpha \text {-domX2 } & 10 & 14 & 3.65 & 5.08 & 0.18 & 0.102 & 4 \times 10^{-4} & 3.3 \times 10^{-7} & -0.025 & 4 \\ \text { olA } \alpha 4 \text {-domX3 } & 8.44 & 11.82 & 3.65 & 5.08 & 0.18 & 0.102 & 4 \times 10^{-4} & 3.3 \times 10^{-7} & -0.025 & 4 \\ \text { olA } \alpha 4 \text {-domY2 } & 8.44 & 11.82 & 3.65 & 5.08 & 0.34 & 0.102 & 4 \times 10^{-4} & 3.3 \times 10^{-7} & -0.025 & 4 \\ \text { olA } \alpha 4-\operatorname{dom} Z 2 & 8.44 & 11.82 & 3.65 & 5.08 & 0.18 & 0.154 & 4 \times 10^{-4} & 3.3 \times 10^{-7} & -0.025 & 4 \\ \text { olA } \alpha 4-\Delta x 2 & 8.44 & 11.82 & 3.65 & 5.08 & 0.18 & 0.108 & 2.8 \times 10^{-4} & 2.3 \times 10^{-7} & -0.025 & 4 \\ \text { olA } \alpha 8 & 8.44 & 11.82 & 3.65 & 5.08 & 0.18 & 0.102 & 4 \times 10^{-4} & 3.3 \times 10^{-7} & -0.025 & 8 \\ \text { olB } 44 & 8.44 & 11.82 & 3.65 & 5.08 & 0.18 & 0.102 & 4 \times 10^{-4} & 3.3 \times 10^{-7} & -0.0295 & 4 \\ \text { olC } \alpha 4 & 8.44 & 11.82 & 3.65 & 5.08 & 0.18 & 0.102 & 4 \times 10^{-4} & 3.3 \times 10^{-7} & 0.01 & 4\end{array}$

TABLE 2. Computational parameters of the simulated cases (see figure 3). Lengths are non-dimensionalized by the stowed chord $\left(c_{s t}\right)$.

Therefore, the other cases have 131 STT total time. The pressure signal is segmented in blocks with $50 \%$ overlap, and a Hanning window is used, resulting in a Strouhal number resolution of $\Delta S t \approx 0.11$. Owing to a mistake in the generation of the output file for case $o l A \alpha 4-\operatorname{dom} X 1$, the initial 63 STT are discarded, and hence the useful time is reduced to $68 \mathrm{STT}$ for this case. As shown in figure 5, very good agreement is found for all spectra, with a relatively smaller amplitude for the first narrowband peak just above $S t=2$ for the case $o l A \alpha 4-\operatorname{dom} X 1$. The remaining of the spectra are virtually the same for all the cases, and the model is deemed to be insensitive to variations of the anechoic layer and streamwise domain extension. Consequently, the smallest domain is used for the simulation of the other slat configurations. The tests show that the low-frequency narrowband peaks are fairly insensitive to the anechoic layer parameters, demonstrating that the approach is able to remove the transient without affecting the mechanism of slat sound generation in the simulations.

All the FW-H computations discussed in this work consider the noise emitted by the slat to an observer at the lower computational boundary, $0.63 c_{s t}$ downstream of the slat trailing edge at $\alpha=0^{\circ}$. This observer position corresponds to the centre of the 


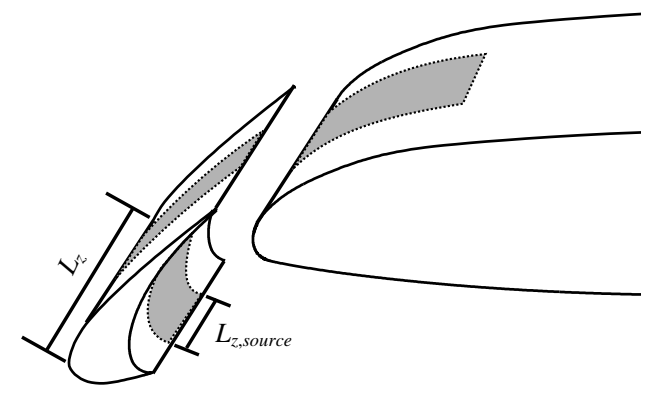

FIGURE 6. Schematic view of the source region used in the Ffowcs Williams-Hawkings analogy computations.

microphone array in the reference experiments. In all cases, the source region adopted comprises the slat surface and the main element's surface around the leading-edge region, as shown in figure 6, and consequently quadrupole terms are neglected. Studies by Lockard \& Choudhari (2009) comparing FW-H models with and without quadrupole source terms show that their effect is negligible on the noise computed up to frequencies corresponding to $S t \times M a=1.7$ for the same geometry studied here. Since the frequency range of our interest lies below $S t \times M a=0.7$, neglecting the quadrupole contribution is justified in our study. To avoid the effect of the periodic boundary condition on far-field noise calculations, only the central half of the spanwise domain is used as FW-H source, i.e. $L_{z \text {,source }}=0.5 L_{z}$ in figure 6 . These FW-H source region and observer position are considered throughout the paper.

The sensitivity of the results to the extent of the computational domain in the spanwise direction is investigated in figure $7(a)$ by comparing results from cases $o l A \alpha 4-\operatorname{dom} X 3$ and olA $\alpha 4-\operatorname{dom} Z 2$, which differ by $51 \%$ in spanwise length. The spectra are corrected to account for the variation of the spanwise dimension of the FH-W analogy integration surface. The amplitude of the peaks of higher frequencies is found to decrease with the spanwise domain extension. Yet, for the dominant peaks, the difference remains smaller than $3 \mathrm{~dB} \mathrm{~Hz}^{-1}$. As a consequence, the sensitivity to spanwise domain extension is considered sufficiently small for the objectives of the current investigation and in the following the smaller domain is used.

The PSD of slat noise for cases olA $\alpha 4-\operatorname{dom} X 3$ and $o l A \alpha 4-\Delta x 2$ are compared to evaluate the simulation's sensitivity to grid refinement (figure $7 b$ ). The effect of the grid refinement on the noise predicted for the configuration olA $\alpha 4$ is restricted to the acoustic power related to some of the low-frequency peaks, and lies within $5 \mathrm{~dB} \mathrm{~Hz}^{-1}$. For the experimental validation of the numerical results, figure $7(b)$ also includes results from the experiments of Pagani et al. (2016), where a complete experimental and numerical comparison is presented. In the processing of the numerical data in figure $7(b)$, the frequency resolution varies along the frequency range considered, in order to match the procedure applied to the experiments. The frequency bin is $\Delta f=50 \mathrm{~Hz}(\Delta S t=0.11)$, for frequencies below $3.3 \mathrm{kHz}(S t=7.26)$, $\Delta S t=0.22$, between $S t=7.26$ and 14.08, $\Delta S t=0.44$ between $S t=14.08$ and 28.16 and $\Delta S t=0.88$, for frequencies above $S t=20.16$. The measurements were carried out with an array of 62 microphones. The background noise was approximately $20 \mathrm{~dB} \mathrm{~Hz}^{-1}$ below the measured slat noise in the frequency range of interest. For 

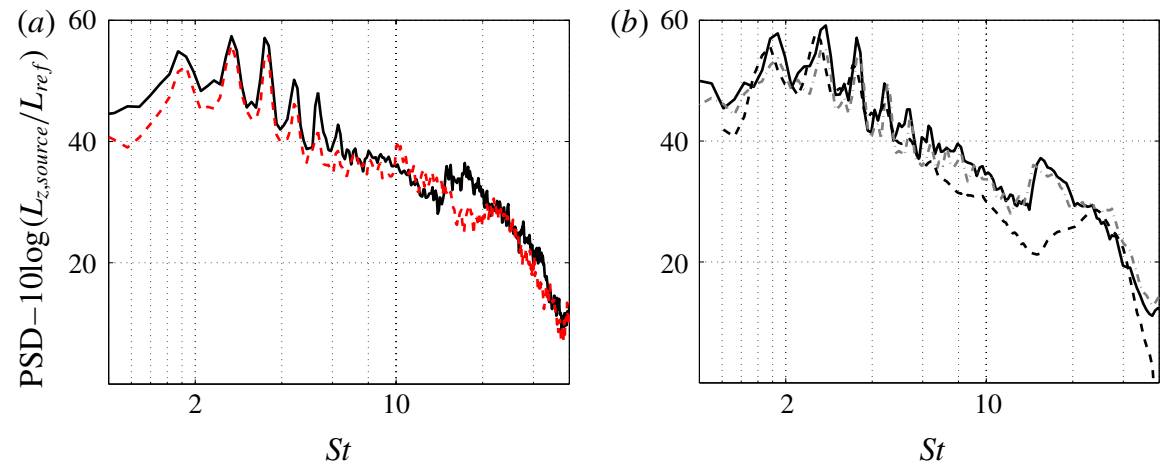

FIGURE 7. (Colour online) PSD of pressure fluctuations at a point corresponding to the centre of the microphone array in the reference experiments computed with Ffowcs Williams-Hawkings (FW-H) analogy. (a) Different spanwise extensions of the computational domain, cases olA $\alpha 4-\operatorname{dom} X 3$ (solid black line) and olA $\alpha 4$-domZ2 (dashed red line). (b) Numerical results with different grid resolutions, olA $\alpha 4-\operatorname{dom} X 3$ (solid black line) and olA $\alpha 4-\Delta x 2$ (dashed grey line) and to experiments by Pagani et al. (2016) (dashed black line).

detailed description of the measurement refer to Pagani et al. (2016) and Pagani et al. (2017). There is a substantial difference between numerical and experimental results in the Strouhal number range from 9 to 20. This is, however, a region with relatively small noise emission and is not the focus of the current investigation.

The frequencies of the narrowband peaks are well predicted by the simulations although the amplitude of some peaks is slightly overpredicted. The agreement between the measured and predicted amplitudes is within $5 \mathrm{~dB} \mathrm{~Hz}^{-1}$. In summary, the narrowband peaks are shown to be fairly independent of the computational parameters, namely, grid and domain. At the same time, the models employed, such as turbulence and acoustic models, are shown to be adequate by the good comparison with the experimental results in the frequency range of interest. In view of that the numerical results are considered a good basis to investigate the dominant mechanism of sound generation in the slat.

The same numerical procedure is adopted for the other three configurations. For all cases, the pressure distribution and the noise spectrum is shown in figures 8 and 9 respectively, together with measurements from Pagani et al. (2016) and Pagani et al. (2017). The greatest disagreement between computation and experiment is observed for case $o l A \alpha 8$. Although the reason for this difference is still not clear, it may be related to excessive suction of the wind tunnel wall boundary layer, which was set based on the main-element spanwise pressure distribution (Amaral et al. 2019). However, the qualitative effect of the angle of attack on the frequency and amplitude of the narrowband peaks is captured by the simulation. For cases olB $\alpha 4$ and $o l C \alpha 4$, the agreement is within $5 \mathrm{~dB} \mathrm{~Hz}^{-1}$. The reduction of the low-frequency slat noise with increasing angle of attack captured by the present simulations agrees with previous results in the literature (Dobrzynski \& Pott-Pollenske 2001; Kolb et al. 2007; Imamura et al. 2009; Amaral et al. 2017). Low-frequency sound amplitude, especially for the narrowband peaks, is reduced as the overlap is increased, to the point that the peaks are not observed for the largest overlap simulated, olC $\alpha 4$. 

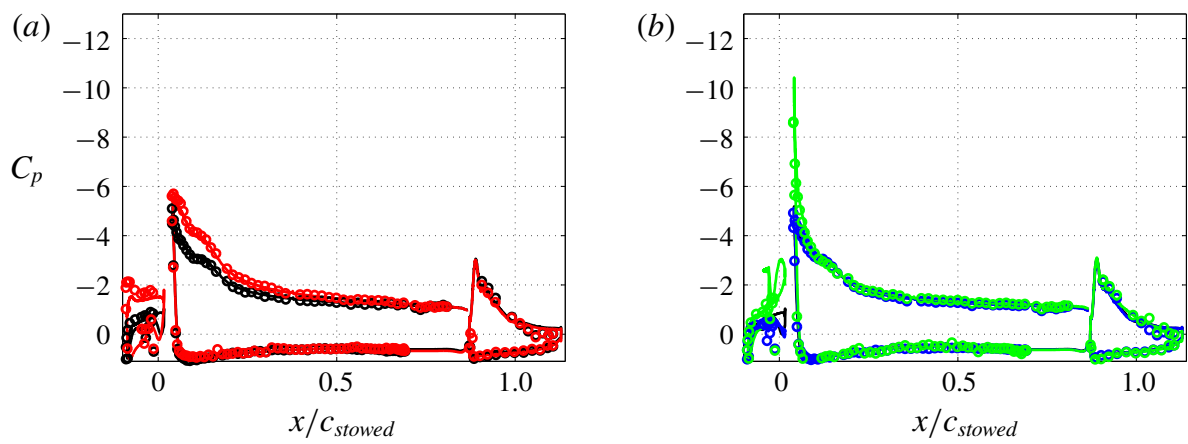

FIGURE 8. (Colour online) Time-averaged pressure coefficient $C_{p}=\left(p-p_{\infty}\right) /\left(0.5 \rho U_{\infty}^{2}\right)$ on the centre plane. (a) Effect of angle of attack on the baseline MD30P30N configuration and (b) effect of slat overlap. Cases olA $\alpha 4$ (black line), olA $\alpha 8$ (red line), olB $\alpha 4$ (blue line) and olC $\alpha 4$ (green line). Experimental results from Pagani et al. (2016) in (a) and Pagani et al. (2017) in (b) are indicated by the symbols.
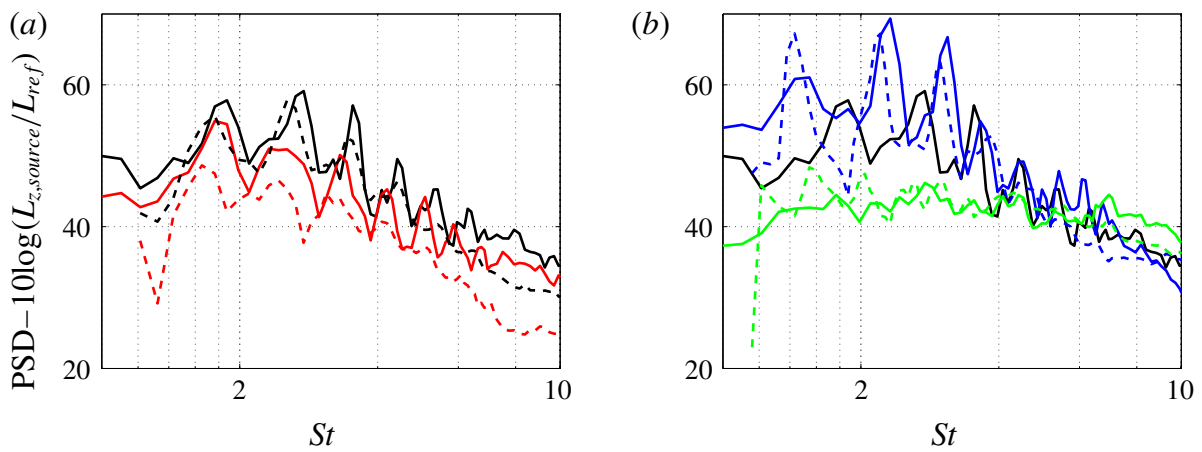

FIGURE 9. (Colour online) PSD of slat noise computed with FW-H analogy for an observer at a point corresponding to the microphone array centre of the reference experiments. (a) Angle of attack effect and (b) slat overlap effect. Cases olA 44 (black line), olA $\alpha 8$ (red line), olB $\alpha 4$ (blue line) and olC $\alpha 4$ (green line). Solid line correspond to present simulations and dashed lines to measurements by Pagani et al. (2016) in (a) and Pagani et al. (2017) in (b).

\section{Proper orthogonal decomposition analysis}

\subsection{POD method}

The proper orthogonal decomposition is a correlation technique that decomposes a given set of empirical realizations, $\left\{\boldsymbol{q}^{i}\right\}$, in an orthonormal, optimal basis $\left\{\boldsymbol{\phi}_{j}\right\}$. The optimality of the delivered basis means that, for a prescribed correlation metric, there is no other orthogonal basis that better represents, on an average sense, the elements $\left\{\boldsymbol{q}^{i}\right\}$ with a given finite number of modes than the POD basis formed by the elements $\left\{\boldsymbol{\phi}_{j}\right\}$. Holmes, Lumley \& Berkooz (1996) presents the method as an appropriate mean to reduce the order of infinite-dimensional dynamic systems. Here, the method is used to identify the large-scale structures or dominant occurrences in the unsteady flow with focus on the turbulent processes generating slat noise. This functionality of the method has been used in the field of aeroacoustics for studies of other flows such as jets 
(Arndt, Long \& Glauser 1997; Freund \& Colonius 2009; Sinha et al. 2014) and open cavity flow (Druault, Gloerfelt \& Mervant 2011).

The snapshot method proposed by Sirovich (1987) is employed here to compute the POD values and functions. Projecting the POD modes onto the basis of flow realizations $\left\{\boldsymbol{q}^{i}\right\}$, we can write

$$
\tilde{\boldsymbol{R}} \boldsymbol{b}_{j}=\overline{\left\langle\boldsymbol{q}^{k}, \boldsymbol{q}^{i}\right\rangle} \boldsymbol{b}_{j}=\lambda_{j} \boldsymbol{b}_{j},
$$

which is an eigenvalue problem whose dimension is the number of realizations, $N_{r}$, and the repeated index does not imply summation. The eigenvalues $\lambda$ are the first $N_{r}$ POD values, while the element $b_{j}^{i}$ of the eigenvector is the projection of the $j$ th POD function onto realization $\boldsymbol{q}^{i}$. Thus, the $j$ th POD function can be recovered as $\sum_{i=1}^{N_{r}} b_{j}^{i} \boldsymbol{q}^{i}$.

POD functions become Fourier modes in the directions for which the realizations are translation invariant (Holmes et al. 1996). Therefore, a Fourier transformation can be performed a priori, and the POD can be applied separately to the transformed realizations for each Fourier mode. In the study described here, the data are homogeneous in the spanwise direction, due to the periodicity condition imposed, and in time, as the developed turbulent flow is assumed to be ergodic. The realizations $\boldsymbol{q}^{i}$ are $z$ - $t$-Fourier transforms of the data, segmented in time blocks, i.e.

$$
\hat{\boldsymbol{q}}(x, y, \beta, \omega)=\frac{1}{T_{b} L_{z}} \int_{T_{b}} \int_{L_{z}} \boldsymbol{q}^{\prime}(x, y, z, t) \mathrm{e}^{-\mathrm{i}[\omega t+\beta z]} \mathrm{d} z \mathrm{~d} t,
$$

where $T_{b}$ is the block length, $\omega$ is the angular frequency, $\beta=2 \pi / \lambda_{z}$ is the spanwise wavenumber, $\lambda_{z}$ is the spanwise wavelength and $\boldsymbol{q}^{\prime}$ is the vector of flow variables after subtraction of the time-spanwise average of each block. Vector-valued flow realizations, $\hat{\boldsymbol{q}}=\{\hat{u}, \hat{v}, \hat{w}, \hat{p}\}$ are considered, where $\hat{u}, \hat{v}$ and $\hat{w}$ are the $z$-t-Fourier transform of the streamwise, vertical and spanwise velocity components, and $\hat{p}$ denotes the transform of the static pressure.

For the POD computations, the LBM simulation data are saved every 64 simulation time steps, which corresponds to approximately 0.01 STT. The time series are segmented in blocks with frequency resolution $\Delta S t=0.11$, resulting in a total of 13 blocks with $50 \%$ overlap. In the spanwise direction, data are saved for 32 stations along the entire simulation domain, with a spacing of approximately 0.021 slat chord. Thus, the non-dimensional spanwise wavenumber resolution is $\Delta \tilde{\beta}=2 \pi c_{\text {slat }} / L_{z}=9.2$.

Different correlation metrics can be written generically as

$$
\left\langle\hat{\boldsymbol{q}}^{i}, \hat{\boldsymbol{q}}^{j}\right\rangle=\int_{\Omega}\left(\hat{u}^{i} \sigma_{u} \hat{u}^{i *}+\hat{v}^{i} \sigma_{v} \hat{v}^{i *}+\hat{w}^{i} \sigma_{w} \hat{w}^{j *}+\hat{p}^{i} \sigma_{p} \hat{p}^{j *}\right) \mathrm{d} V,
$$

where $\boldsymbol{\sigma}(\boldsymbol{x})=\left[\sigma_{u}, \sigma_{v}, \sigma_{w}, \sigma_{p}\right]$ are spatially varying weighting factors defined for each variable.

Two correlation metrics are used in the present POD computations. The first one corresponds to the conventional turbulent kinetic energy (TKE) metric, integrated over a portion of the domain containing the slat and the leading edge of the main element (in black in figure 10). For the TKE correlation metric, the weighting factor is $\boldsymbol{\sigma}(\boldsymbol{x})=$ $[1,1,1,0]$ in this region and zero elsewhere. The second metric used consists of the integration of the pressure over a horizontal plane below the airfoil, far from the noise source region. The corresponding weighting factor is $\boldsymbol{\sigma}(\boldsymbol{x})=[0,0,0,1]$ at the horizontal plane shown in figure 10 and zero elsewhere. 


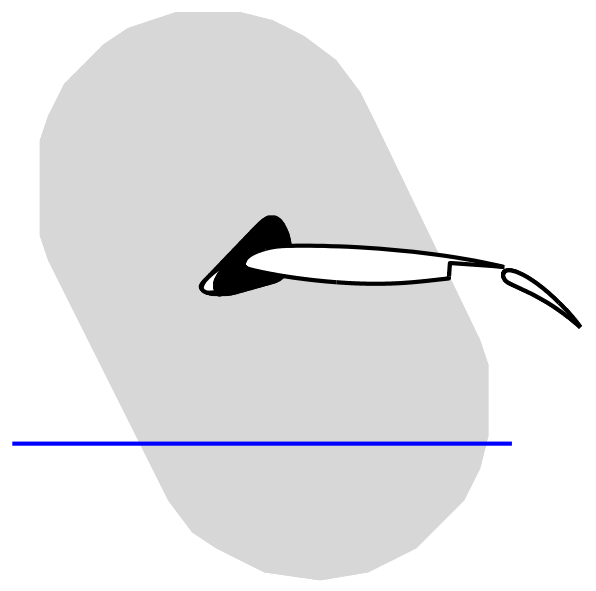

FIgURE 10. (Colour online) Regions with data used in the proper orthogonal decomposition (POD) computations. Noise source region comprising the slat and main element leading edge (in black), plane in the far field (horizontal line) and region including turbulence and sound fields extracted for case olA 4 (in grey).

The methodology applied is inspired by Freund \& Colonius (2009), who compare different correlation metrics for the investigation of the turbulent structures in the flow of a 0.9 Mach number jet. The different metrics result in structures of different shapes identified in the respective POD modes. The turbulent kinetic energy metric identifies the most energetic flow structures within the slat cove flow, which are not necessarily related to the sound production. On the other hand, the metric based on the far-field pressure recovers the flow structures most correlated to the emitted noise, which do not necessarily corresponds to the most energetic ones, but are more likely to be related to the noise sources.

The region around the slat where data are stored for POD computations comprises part of the grid refinement levels 7,6 and 5 (see figure 4). The data from grid refinement level 7 (which has a resolution of approximately $2.7 \times 10^{-3}$ times the slat chord) are averaged over groups of eight cells $(2 \times 2 \times 2$, considering the three coordinate directions) resulting in larger cells with edge length equal to $5.3 \times 10^{-3}$ times the slat chord, hereinafter referred to as measurement cells. On the horizontal far-field plane (figure 10), the POD data points are separated from each other approximately 0.021 of the slat chord in the streamwise direction, which is the LBM simulation grid resolution for that region. As in all analyses the flow realizations comprise data both in the slat region and in the far-field plane, hence the POD modes represent flow features in the two regions. Data from an additional flow region, shown in grey in figure 10, are stored for the case olA $\alpha 4$. The aim is to investigate the flow field fluctuations in the region containing both the turbulent and sound fields which are correlated to the coherent pressure fluctuations in the far-field plane. For the two metrics considered, the weighting factor in the grey area is zero and the spatial linear resolution of the data stored is approximately 0.021 times the slat chord for this region.

\subsection{Large-scale structures}

Normalized POD values corresponding to TKE metric for case olA $\alpha 4$ are shown in figure 11 as a function of non-dimensional spanwise wavenumber $\left(\tilde{\beta}=2 \pi c_{\text {slat }} / \lambda_{z}\right.$, 
(a)
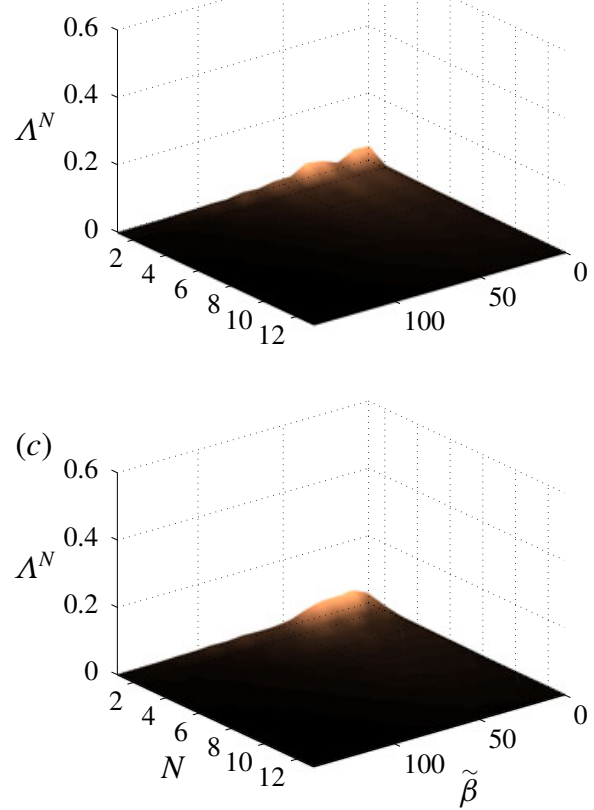

(b)

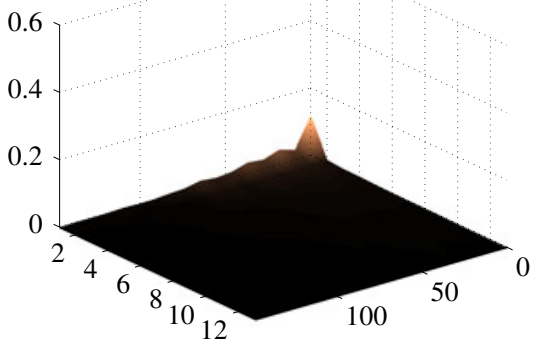

(d)

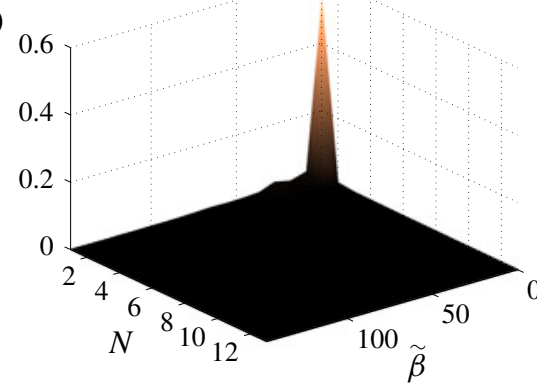

FIGURE 11. (Colour online) Turbulent-kinetic-energy-based normalized POD values for the case $o l A \alpha 4$ at selected frequencies: (a) $S t=1.88$ (narrowband peak), (b) $S t=2.76$ (narrowband peak), (c) $S t=2.98$ (broadband) and $(d) S t=3.53$ (narrowband peak).

where $\lambda_{z}$ is the spanwise wavelength) and mode number $(N)$. Results for four frequencies are presented, three corresponding to narrowband peaks in the noise spectrum $(S t=1.88,2.76$ and 3.53) and one corresponding to broadband noise $(S t=2.98)$. The POD values for the first narrowband peak, figure 11(a), and for the broadband noise frequency, figure 11(c), are significantly spread over the spanwise wavenumber axis, revealing a highly three-dimensional character of the flow structures. On the other hand, the first mode with $\tilde{\beta}=0$ dominates the spectra for the second and third peaks, figures $11(b)$ and $11(d)$ respectively, indicating that the first two-dimensional (2-D) POD modes capture a substantial portion (approximately $10 \%$ and $54 \%$ respectively) of the turbulent kinetic energy associated with these narrowband peak frequencies.

Figure 12 shows the real part of the velocity components of the leading 2-D TKEbased POD mode at $S t=3.53$, a frequency of a narrowband peak. It is seen that the leading two-dimensional modes are localized along the mixing-layer path. The shape of this mode's $x$ - and $y$-velocity components strongly relates it to the $\mathrm{K}-\mathrm{H}$ instability. An analogous picture is obtained for the first 2-D POD mode for all other frequencies. Figure 13 shows the real part of the velocity components of dominant POD mode for the broadband noise frequency of figure 11(c). The mode seems to represent spanwise modulated $\mathrm{K}-\mathrm{H}$ vortices and $3-\mathrm{D}$ fluctuations in the slat wake. The real part of the velocity components of the first $(S t, \tilde{\beta})=(1.88,36.6)$ TKE-based POD mode corresponding to a peak frequency is shown in figure 14. It captures an amount of energy comparable to the first 2-D mode for the same frequency and reveals structures in the slat that represent predominantly streamwise stretched vortices being ejected through the gap (Imamura et al. 2008; Deck \& Laraufie 2013). 
(a)

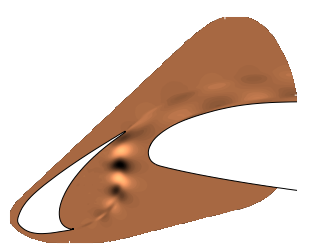

(b)

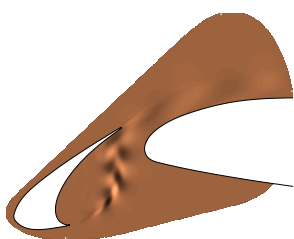

(c)

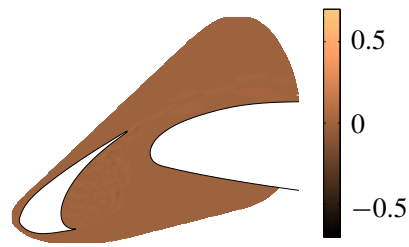

FIGURE 12. (Colour online) Real part of the velocity components of the turbulent-kineticenergy-based POD mode for $(S t, \tilde{\beta})=(3.53,0)$ (narrowband peak) from case olA $\alpha 4$. (a) Streamwise, (b) vertical and (c) spanwise components.

(a)

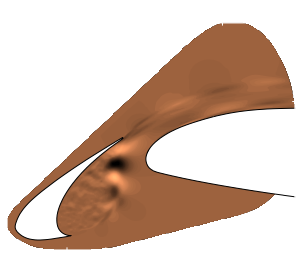

(b)

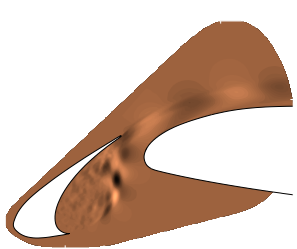

(c)

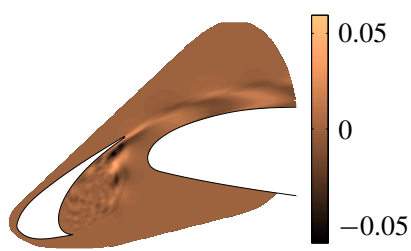

FIGURE 13. (Colour online) Real part of the velocity components of the turbulent-kineticenergy-based POD mode for $(S t, \tilde{\beta})=(2.98,9.2)$ (broadband noise) from case olA $\alpha 4$. (a) Streamwise, $(b)$ vertical and $(c)$ spanwise components.

(a)

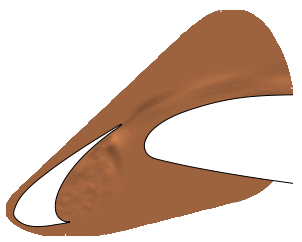

(b)

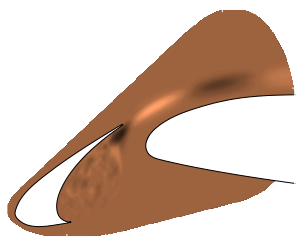

$(c)$

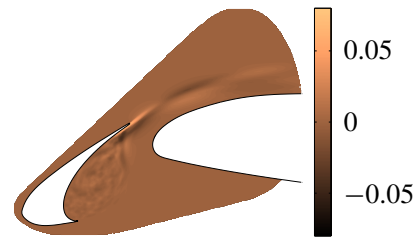

FIGURE 14. (Colour online) Real part of the velocity components of the turbulent-kineticenergy-based POD mode for $(S t, \tilde{\beta})=(1.88,36.6)$ (narrowband peak) from case olA $\alpha 4$. (a) Streamwise, $(b)$ vertical and $(c)$ spanwise components.

POD values based on the acoustic pressure metric applied to the case olA $\alpha 4$ are given in figure 15, showing the dominance of the first two-dimensional mode, both for frequencies associated with narrowband peaks and for frequencies of broadband noise. The acoustic 'energy' captured by the leading 2-D POD is at least $70 \%$ of the total in all cases analysed, being higher than $90 \%$ for some narrowband peak frequencies, which indicates that the far-field pressure fluctuations below the airfoil are strongly coherent over the span. The real part of the velocity components of the leading POD mode based on the acoustic pressure for $S t=3.53$ (a narrowband peak frequency) are shown in figure 16. Similar to the dominant TKE-based POD mode, for this frequency, the dominant acoustic-based POD mode resembles the $\mathrm{K}-\mathrm{H}$ vortices. Kelvin-Helmholtz vortices were found to dominate the first 2-D acoustic-pressurebased POD modes for the other frequencies as well.

The POD analysis was extended to the other configurations. For the cases olA $\alpha 8$ and $o l B \alpha 4$, the TKE-based POD spectra are dominated by the first 2-D mode at the frequencies of the narrowband peaks, while the corresponding spectra were dominated by three-dimensional POD modes at broadband noise frequencies. Although case 
(a)

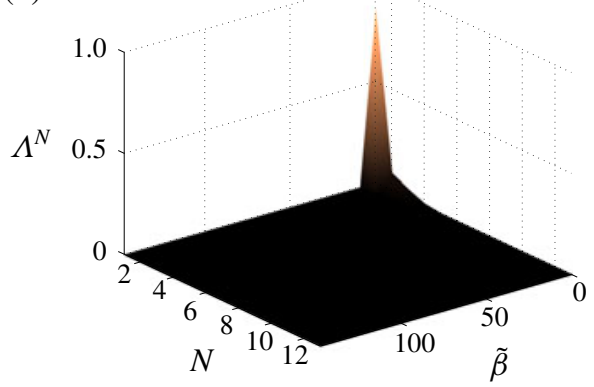

(c)

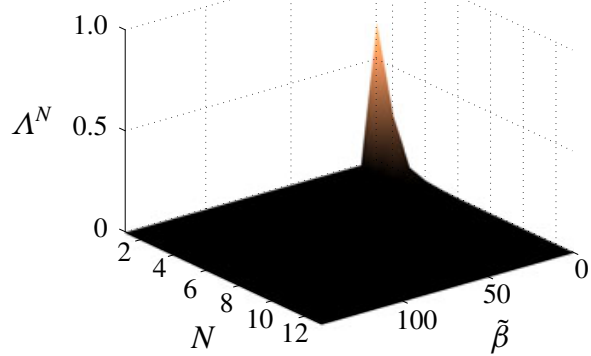

(b)

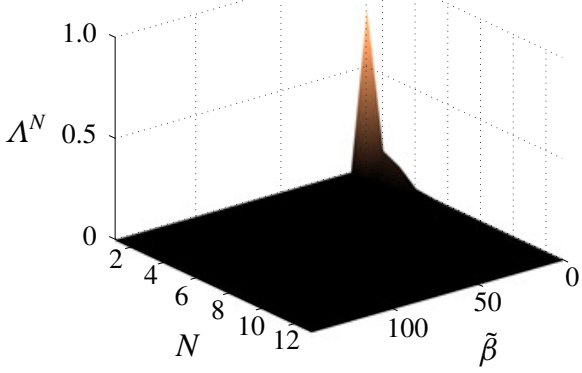

(d)

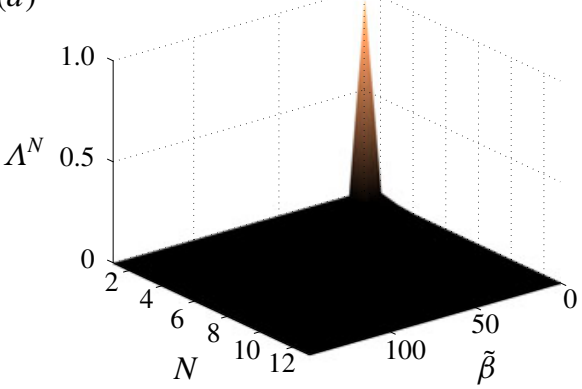

FIgURE 15. (Colour online) Acoustic-based normalized POD values from case olA $\alpha 4$ at selected frequencies: (a) $S t=1.88$ (narrowband peak), (b) $S t=2.76$ (narrowband peak), (c) $S t=2.98$ (broadband) and (d) $S t=3.53$ (narrowband peak).

(a)

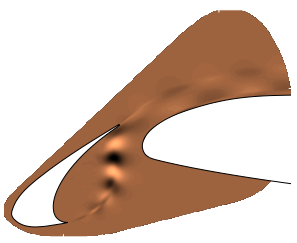

(b)

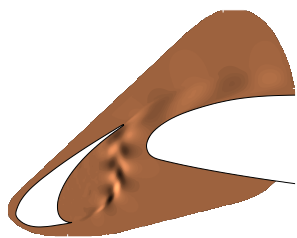

(c)

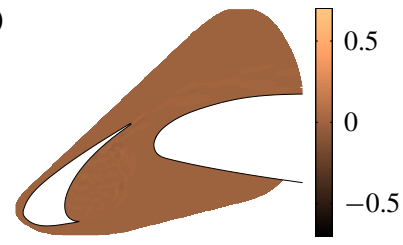

FIgURE 16. (Colour online) Real part of the velocity components of the acoustic-based POD mode for $(S t, \tilde{\beta})=(3.53,0)$ (narrowband peak) from case olA $\alpha 4$. (a) Streamwise, (b) vertical and $(c)$ spanwise components.

olC $\alpha 4$ does not present the peaks, its TKE-based POD spectrum has a 2-D dominant mode for some of the frequencies analysed. Moreover, the 2-D TKE-based POD modes for these three other cases are dominated by structures resembling the $\mathrm{K}-\mathrm{H}$ waves in the mixing layer, as shown in figure 17, while 3-D TKE-based POD modes represent energetic structures in the mixing layer, and also in the slat gap and wake (figure 18). Also in line with the observations for case olA $\alpha 4$, spanwise-coherent pressure fluctuations dominate in the far field for the other conditions tested. For these other three cases, the first 2-D acoustic-pressure-based POD mode captures more than $60 \%$ of the energy of the far-field pressure fluctuations, and resembles $\mathrm{K}-\mathrm{H}$ structures in the mixing layer regardless of the frequency corresponding to narrowband peak or broadband noise. 
(a)

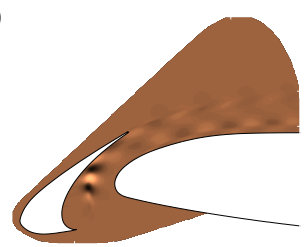

(b)

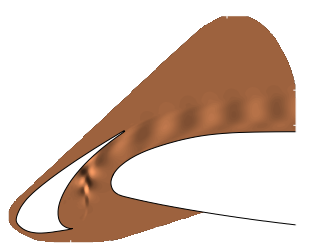

(c)

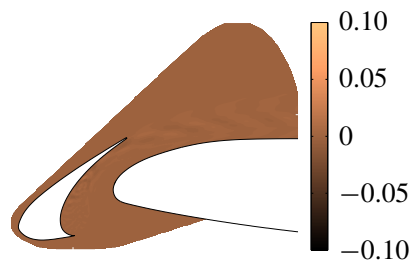

FIGURE 17. (Colour online) Real part of the velocity components of the turbulent-kineticenergy-based POD mode for $(S t, \tilde{\beta})=(5.63,0)$ from case olC $\alpha 4$. (a) Streamwise, $(b)$ vertical and $(c)$ spanwise components.

(a)

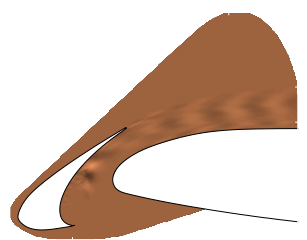

(b)

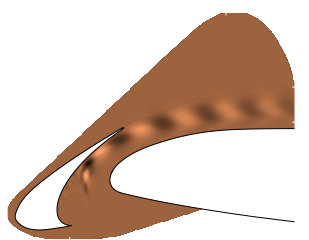

(c)

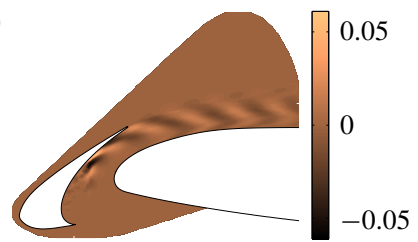

FIGURE 18. (Colour online) Real part of the velocity components of the turbulent-kineticenergy-based POD mode for $(S t, \tilde{\beta})=(5.63,36.8)$ from case olC $\alpha 4$. (a) Streamwise, $(b)$ vertical and $(c)$ spanwise components.

In summary, the POD analysis showed that for all frequencies analysed the slat noise is correlated to spanwise aligned structures even if they are not the most energetic ones. Although our analysis so far does not reveal the frequency selection mechanism, it is consistent with a feedback loop: the sound emitted at the slat's trailing edge being fundamentally two-dimensional triggers spanwise-coherent $\mathrm{K}-\mathrm{H}$ structures at the cusp. At narrowband peaks the sound is loud and leads to a prevalence of spanwise-coherent structures in the flow field. Off the peaks they become considerably more three-dimensional.

\section{Feedback mechanism}

\subsection{Evidences of the feedback mechanism}

Terracol et al. (2016) proposes a feedback loop model for the generation of the narrowband peaks in the slat noise spectrum, which is inspired in the classical Rossiter (1966) model for the generation of acoustic tones by an open cavity flow: a pressure wave introduces a disturbance in the mixing layer at the slat cusp; this disturbance is amplified due to the mixing-layer instability as it is advected towards the reattachment point; then the large amplitude disturbance impinges on the slat cove surface generating a new pressure wave front; finally, this new pressure disturbance travels upstream exciting the mixing-layer instability at the cusp. Denoting the distance travelled by the mixing-layer disturbance from the cusp to the reattachment point by $L_{v}$, the distance travelled by the pressure wave (assuming a straight path from the reattachment to the cusp) by $L_{a}$, the mixing-layer disturbance phase velocity by $U_{v}$, and neglecting convective effects on the acoustic propagation since the Mach number is too low, this model results in a series of discrete frequencies

$$
S t_{n, \text { Terracol }}=n \frac{c_{\text {slat }}}{U_{\infty}}\left(\frac{L_{v}}{U_{v}}+\frac{L_{a}}{c_{\infty}}\right)^{-1},
$$

where $n=1,2,3, \ldots$, and $c_{\infty}$ is the free-stream sound speed. 
(a)

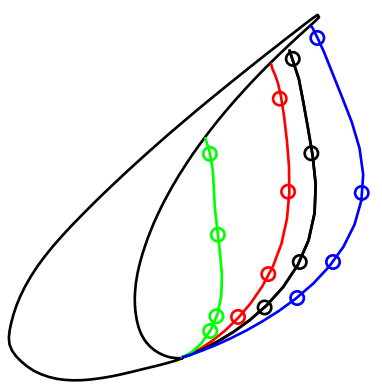

(b)

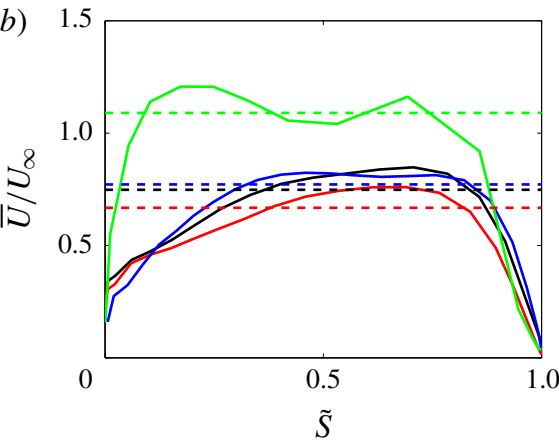

FIgURE 19. (Colour online) (a) Time-averaged mixing-layer path with indication of four stages of the mixing-layer disturbance evolution and $(b)$ time-averaged velocity along the mixing layer (horizontal lines represent the mean velocity between $\tilde{S} \approx 0.15$ and 0.85 ). Black: case $o l A \alpha 4$, red: olA $\alpha 8$, blue: olB $\alpha 4$ and green: olC $\alpha 4$.

$\begin{array}{lcccc} & \text { olA } \alpha 4 & \text { olA } \alpha 8 & \text { olB } \alpha 4 & \text { olC } \alpha 4 \\ L_{v} / c_{\text {slat }} & 0.84 & 0.77 & 0.97 & 0.52 \\ L_{a} / c_{\text {slat }} & 0.74 & 0.68 & 0.79 & 0.49 \\ U_{v} / U_{\infty} & 0.75 & 0.67 & 0.77 & 1.09\end{array}$

TABLE 3. Normalized time-averaged flow properties of the slat mixing layer. $U_{v}$ is the estimated phase velocity of the mixing-layer disturbance.

The values of $L_{v}, L_{a}$ and $U_{v}$ are estimated from the simulations based on the time-averaged mixing-layer path (figure 19a) and time-averaged velocity along it $\bar{U}$ (figure $19 b$ ), where $\tilde{S}=S / L_{v}$ and $S$ is the distance to the cusp along the mixing-layer path. As opposed to Terracol et al. (2016), not all our cases presented a plateau in the $\bar{U}$ distribution. To circumvent this difficulty $U_{v}$ is estimated as the averaged velocity between $\tilde{S} \approx 0.15$ and $\tilde{S} \approx 0.85$, which is an interval of mild acceleration in cases $o l A \alpha 4$ and $o l A \alpha 8$ and of the plateau in cases olB $\alpha 4$ and $o l C \alpha 4$. The normalized values of $L_{v}, L_{a}$ and $U_{v}$ are listed in table 3 and the peak frequencies predicted from (4.1) are listed in table 4 and compared to the peak frequencies of the spectra computed from the simulation data (figure 9). For the cases where the peaks are observed, the predicted frequencies are in fair agreement with the peak frequencies computed from the FW-H data, comparable to that achieved by Deck \& Laraufie (2013) using a similar methodology.

It must be said, however, that since the velocity changes abruptly outside the interval between 15 and $85 \%$, the estimation of $U_{v}$ is highly sensitive to broader limits. Moreover, although Terracol's model is able to provide fairly accurate predictions of the narrowband peaks observed in their slat noise spectrum as well as for the four cases simulated here, it is difficult to reconcile this model with the evidence that the slat trailing edge is the acoustic emission spot, as suggested by the approximate $U_{\infty}^{5}$ velocity scaling law (Dobrzynski et al. 1998) and the spatial directivity pattern (Lockard \& Choudhari 2009; Ewert et al. 2010). Furthermore, as indicated in figure 17, the dynamics of the 2-D mixing-layer structures for the case olC $\alpha 4$ is similar to the other cases both qualitatively and quantitatively. Therefore, 


$\begin{array}{ccccccc} & & n=1 & 2 & 3 & 4 & 5 \\ \text { olA } \alpha 4 & \text { LBM + FW-H } & & 1.88 & 2.76 & 3.53 & 4.41 \\ & S t_{n, \text { Terracol }} & 0.83 & 1.67 & 2.50 & 3.33 & 4.16 \\ & S t_{n} & 1.04 & 1.87 & 2.70 & 3.53 & 4.36 \\ \text { olA } \alpha 8 & \mathrm{LBM}+\mathrm{FW}-\mathrm{H} & & 1.88 & 2.54 & 3.31 & 4.19 \\ & S t_{n, \text { Terracol }} & 0.82 & 1.63 & 2.45 & 3.26 & 4.08 \\ & S t_{n} & 0.96 & 1.73 & 2.49 & 3.26 & 4.03 \\ \text { olB } \alpha 4 & \mathrm{LBM}+\mathrm{FW}-\mathrm{H} & & 1.55 & 2.32 & 3.09 & 3.64 \\ & S t_{n, \text { Terracol }} & 0.75 & 1.50 & 2.25 & 3.00 & 3.75 \\ & S t_{n} & 0.92 & 1.66 & 2.40 & 3.14 & 3.87 \\ \text { olC } \alpha 4 & \mathrm{LBM}+\mathrm{FW}-\mathrm{H} & & \mathrm{X} & \mathrm{X} & \mathrm{X} & \mathrm{X} \\ & S t_{n, \text { Terracol }} & 2.65 & 5.30 & 7.94 & 10.59 & 13.24 \\ & S t_{n} & 1.62 & 2.91 & 4.20 & 5.49 & 6.78\end{array}$

TABLE 4. Discrete peak Strouhal numbers. Comparison between values computed by the FW-H algorithm applied to the LBM computations (denoted by LBM + FW-H), values predicted by the formula $S t_{n, \text { Terracol }}$ (4.1) and the values predicted by (4.5).

there is no apparent reason for the complete attenuation of the narrowband peaks for that case according to this model.

Figure 20 shows contour plots of the magnitude of the pressure component of the dominant acoustic-based POD mode, indicating the trajectory of the $\mathrm{K}-\mathrm{H}$ vortices within the cove. The white line marks the maximum amplitude of the POD structures and gives an indication of the vortex path. The red dashed line is the dividing streamline linking the cusp to the reattachment point (shown in figure 19a). It is observed that, for all cases, the vortices travel along the dividing streamline for a long length, but divert from it close the reattachment point. This suggests a different path for the feedback loop concept, which is described schematically in figure 21: (i) at a reference time $t=0$, the mixing layer is disturbed at the slat cusp; (ii) the disturbance amplifies as it travels along the mixing-layer path; (iii) at $t=T_{h}$, a sound wave front is emitted as the disturbance interacts with the slat trailing edge after travelling the mixing-layer length $L_{v}^{\prime}$ plus a distance $\Lambda$, which accounts for a possible delay between the passage of the mixing-layer disturbance at the trajectory point closest to the trailing edge and the acoustic wave emission (Such phase difference is also accounted for by Rossiter (1966) for open cavities); (iv) the sound wave travels downstream at approximately the free-stream sound speed $c_{\infty}$ and reaches the slat cusp introducing a new disturbance in the mixing layer at $t=T_{h}+L_{a}^{\prime} / c_{\infty}$, where $L_{a}^{\prime}$ is the distance between the slat trailing edge and the cusp. The coordinate $S^{\prime}$ starts from the cusp along the mixing-layer path, goes through the trailing edge and back to the cusp along a straight line (acoustic path). This loop concept circumvents the difficulties raised in last paragraph and results in discrete peak frequencies given by

$$
S t_{n}=n \frac{c_{\text {slat }}}{U_{\infty}}\left(T_{h}+\frac{L_{a}^{\prime}}{c_{\infty}}\right)^{-1} .
$$

It is admitted that, in view of long acoustic wavelength, it can be difficult to distinguish a source at the trailing edge from one at the reattachment point. However, the results discussed hereinafter provide evidence of this distinction. 
(a)

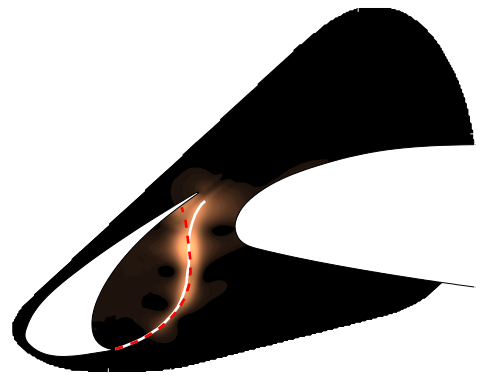

(c)

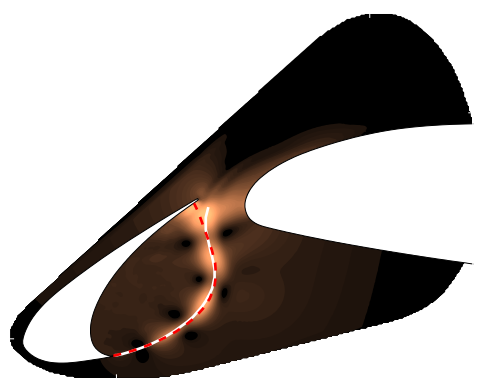

(b)

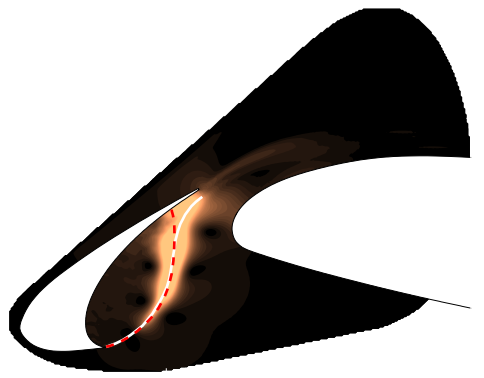

$(d)$

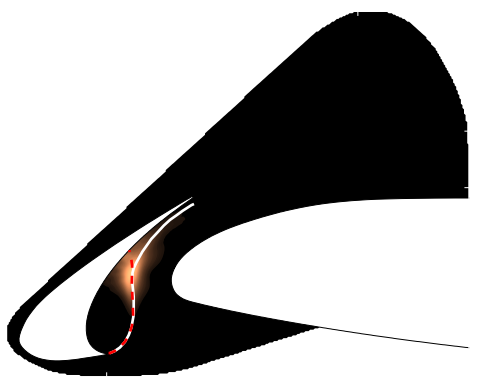

FIgURE 20. (Colour online) Path of the mixing-layer disturbances (solid line) estimated from the amplitude of the pressure component of the dominant acoustic-based POD mode (colour plot) for cases $(a)$ olA $\alpha 4,(b)$ olA $\alpha 8,(c)$ olB $\alpha 4$ and $(d)$ olC $\alpha 4$. The time-averaged streamline from figure $19(a)$ is shown for comparison (dashed lines).
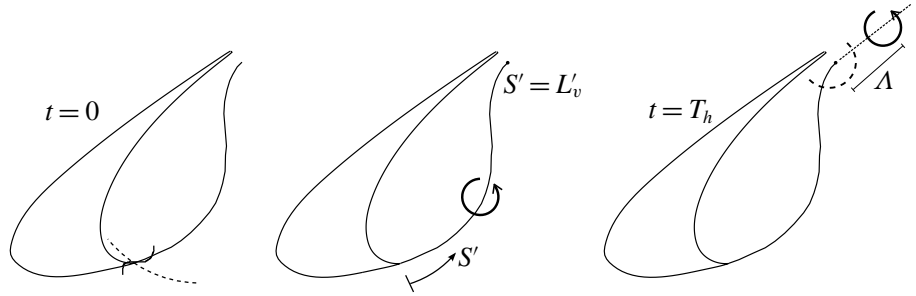

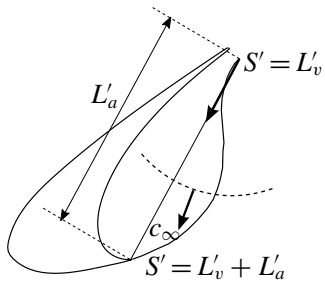

FIGURE 21. Schematic representation of the Rossiter-like feedback loop.

In order to search for confirmation of this feedback loop, the temporal evolution of the dominant acoustic-based POD modes,

$$
\tilde{\boldsymbol{\phi}}(x, y, \tilde{t} ; S t)=\operatorname{Re}\left\{\hat{\boldsymbol{\phi}}_{1}(x, y ; S t, \tilde{\beta}=0) \mathrm{e}^{\mathrm{i} 2 \pi \tilde{t}}\right\},
$$

is analysed, where $\hat{\boldsymbol{\phi}}_{1}(x, y ; S t ; \tilde{\beta}=0)$ is the first two-dimensional acoustic-based POD mode at $S t$ and $\tilde{t}=t U_{\infty} S t / c_{\text {slat }}$ is the non-dimensional time.

Figure 22 shows the pressure component of $\tilde{\boldsymbol{\phi}}(x, y, \tilde{t} ; 3.53)$ for selected times based on case $o l A \alpha 4$. The left-hand side of the figure shows the extended region of the POD mode, where sound waves emanating from the slat cove can be identified, and the right-hand side shows a detailed view of the slat cove. The zoomed-in figure shows, for $\tilde{t}=0$, a negative pressure mixing-layer disturbance reaching the reattachment point region, while in the open view, a compression wave is seen leaving the slat cove downwards. The interaction of the negative pressure mixing-layer disturbance and the 
(a)

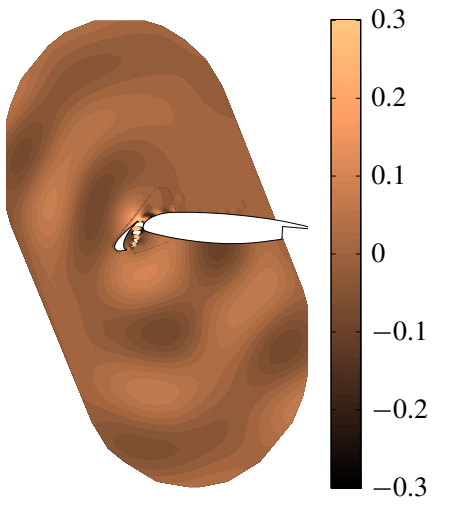

(b)

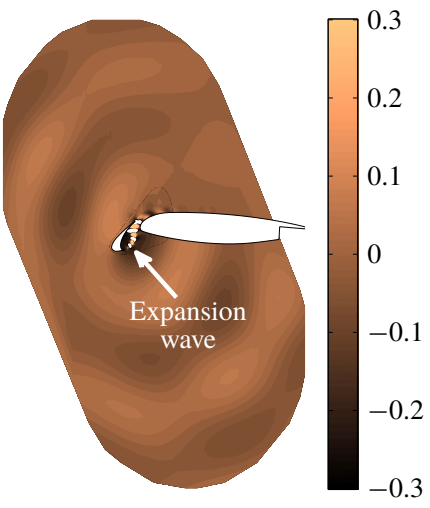

(c)

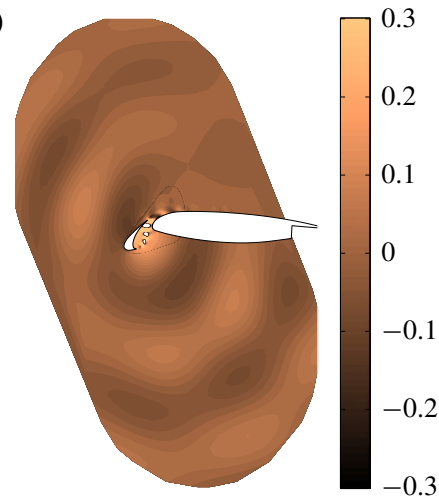

Negative pressure
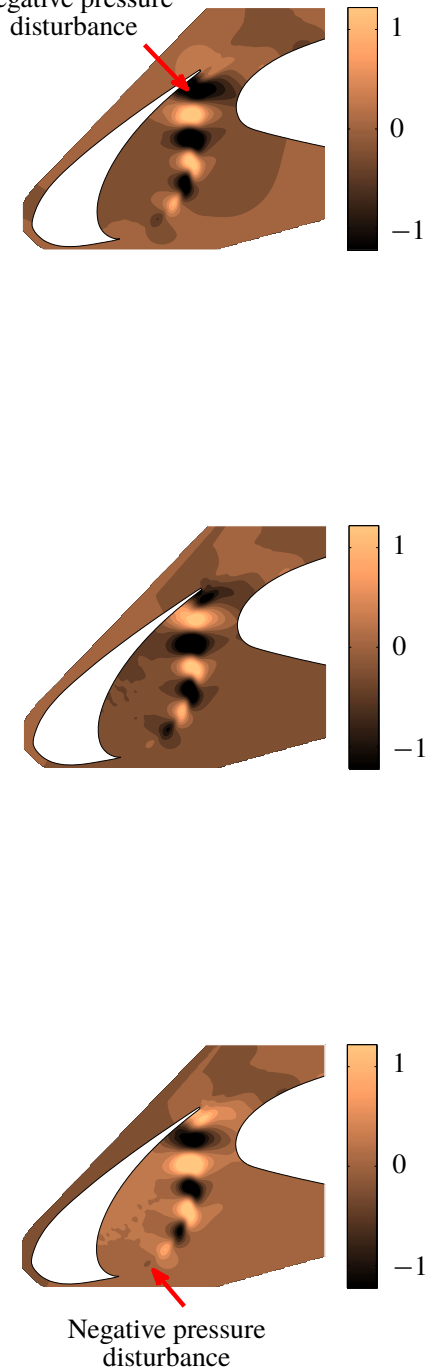

FIgURE 22. (Colour online) Real part of the evolution of the dominant acoustic-based POD mode for $S t=3.53(\tilde{\phi}(x, y, \tilde{t}, S t=3.53 ; \tilde{\beta}=0))$ from case olA $\alpha 4$ at selected times, where $\tilde{t}=t U_{\infty} S t / c_{\text {slat }}$ is the non-dimensional time. (a) $\tilde{t}=0,(b) \tilde{t}=5 / 18$ and $(c) \tilde{t}=3 / 4$.

slat surface generates an expansion wave which dominates the slat cove region at $\tilde{t}=5 / 18$, as seen in the open view. Simultaneously, the pressure at the slat cusp reaches its most negative value along the loop. At $\tilde{t}=3 / 4$, a negative pressure disturbance originates close to the cusp and the expansion wave has left the cove region travelling downwards. The series of events shown in figure 22 is consistent with the acoustic wave fronts leaving the slat cove downwards being generated by the 
interaction of the mixing-layer disturbances with the slat trailing edge. It also reveals a directivity and phase pattern that resembles a dipole at the slat trailing edge.

Figure 23 shows, as function of $\tilde{t}$ and $\tilde{S}^{\prime}=S^{\prime} / L_{v}^{\prime}$ (see figure 21) during six periods, the spatio-temporal evolution of the pressure fluctuation corresponding to the leading acoustic pressure POD mode for selected narrowband peak frequencies, for cases olA $\alpha 4, o l A \alpha 8$ and $o l B \alpha 4(S t=3.53,3.31$ and 3.09, respectively) and for the frequency $S t=5.63$ for the case olC $\alpha 4$. The left portion of the diagrams $\left(\tilde{S}^{\prime}<1\right)$ shows the journey of the $\mathrm{K}-\mathrm{H}$ structures from the cusp to the slat trailing edge. The solid white lines represent the phase evolution of the vortices. The disturbance speed is approximately constant over most of the trajectory, but at the beginning the amplitudes are very small and the advection speed cannot be accurately defined. The right portion represents the acoustic wave journey, i.e. the straight line connecting the ends of the hydrodynamic paths in figure 21. For $\tilde{S}>1$ the phase speed is equal to $c_{\infty}$. From $\tilde{S}=1$ to $\tilde{S} \approx 1.2$ the mode is dominated by the high amplitude $\mathrm{K}-\mathrm{H}$ structures being advected towards the gap and, therefore, do not correspond to the acoustic pressure wave. For the largest portion of the trajectory, the free-stream sound speed is a good representation of the pressure disturbance speed. For all cases, the pressure wave has a $\pi / 2$ phase shift relative to the mixing-layer disturbance at the emission spot.

The diagrams strongly indicate that the disturbances in the mixing layer are introduced by pressure waves which in turn are generated by the interaction of the mixing-layer structures with the slat surface, characterizing a feedback loop. Additionally, the diagrams show that the narrowband peaks at $S t=3.53,3.31$ and 3.09 for cases $o l A \alpha 4, o l A \alpha 8$ and $o l B \alpha 4$ respectively correspond to the fourth Rossiter-like mode: closure of the feedback loop by a particular $\mathrm{K}-\mathrm{H}$ structure, as illustrated by the solid white lines in figure 23, requires four fundamental periods of its corresponding frequency. This is consistent with the observations on the noise spectra because, as can be inferred from table 4, the frequency shift between the narrowband peaks is approximately a quarter of the frequency of the modes shown in figure 23 . It is possible to observe a consistent cycle even in case olC $\alpha 4$, although the acoustic wave evolution looks severely distorted and ill defined.

The dashed white lines in figure 23 represent the averaged phase velocity of the mixing-layer structures in their trajectory from the cusp to the trailing edge. These velocities are slightly higher than the ones in table 3, except for the case olC $\alpha 4$, for which the velocity is significantly higher. Adopting a simplification of uniform mixinglayer disturbance phase velocity equal to the ones represented by the dashed lines, and given the observed phase shift of $\pi / 2$, one can write

$$
T_{h}=\frac{L_{v}^{\prime}}{V}+\frac{\Lambda}{V}=\frac{L_{v}^{\prime}}{V}-\frac{c_{\text {slat }}}{4 U_{\infty} S t_{n}} .
$$

Substituting in (4.2),

$$
S t_{n}=\frac{4 n+1}{4} \times \frac{c_{\text {slat }}}{U_{\infty}} \times \frac{V}{L_{v}^{\prime}+\frac{V}{c_{\infty}} L_{a}^{\prime}} .
$$

Table 4 also presents the narrowband peak frequency predicted by this equation and shows, in general, an improvement comparing to the predictions based on Terracol's formula. 
(a)

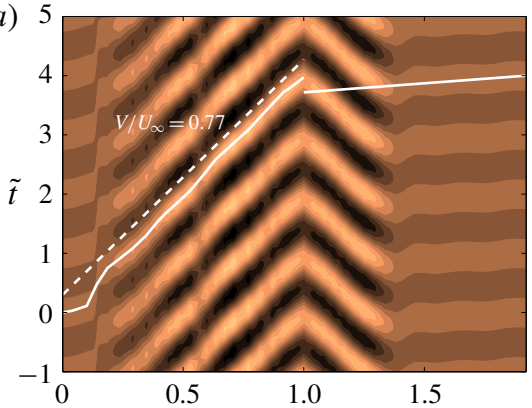

(c)

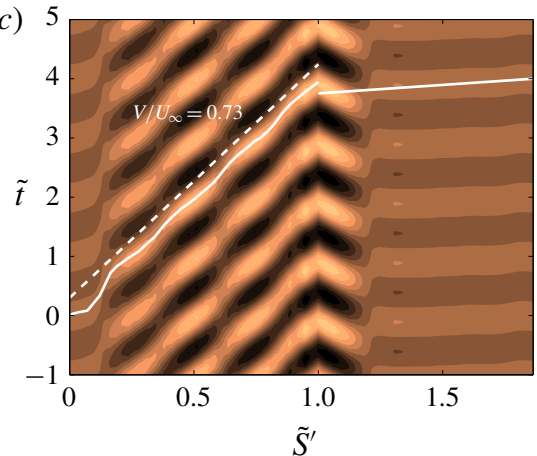

(b)

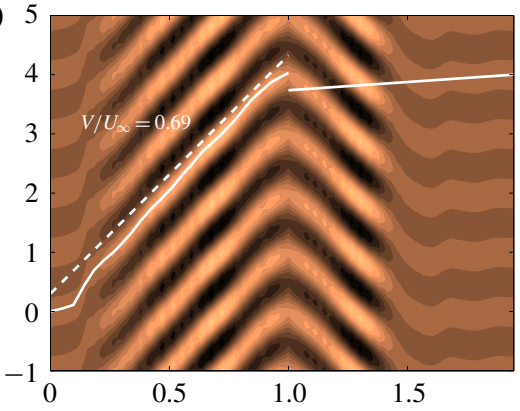

(d)

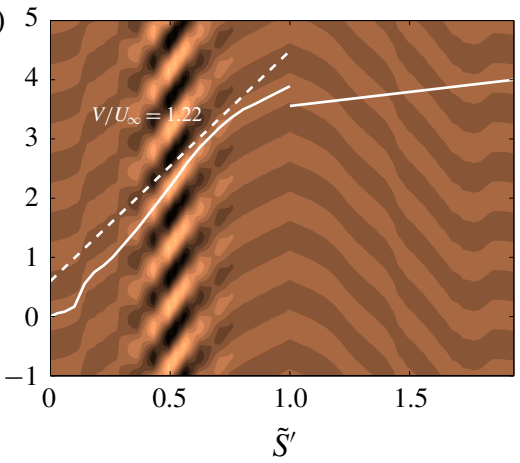

FIgURE 23. (Colour online) Spatio-temporal evolution of the pressure component of dominant acoustic POD mode for narrowband peak frequencies: (a) $S t=3.53$ for the case $o l A \alpha 4$, (b) $S t=3.31$ for the case $o l A \alpha 8$ (c) $S t=3.09$ for the case olB $\alpha 4$ and $(d) S t=5.63$ for the case olC $\alpha 4$ along $\tilde{S}^{\prime}=S^{\prime} / L_{v}^{\prime}$ (see figure 21 ). White solid lines represent phase evolutions where the phase speed is equal to $c_{\infty}$ for $\tilde{S}^{\prime}>1$ and follow maxima of the POD mode along the mixing-layer path.

\subsection{Strength of the feedback mechanism}

These results evidence the frequency selection mechanism unequivocally. However, they do not indicate what conditions enhance or prevent sound emission. In Rossiter's cavity model, the magnitude of a mode is primarily a result of the amplification of the disturbances along the mixing layer (Rowley, Colonius \& Basu 2002; Sun et al. 2017). Figure 24 shows spectra of the streamwise velocity fluctuations at four points representing four stages of evolution of the mixing-layer disturbances discussed by Pagani et al. (2016). Each spectrum is the average of the windowed blocks and the 32 points equally spaced in the spanwise direction. The location of the representative points is indicated in figure $19(a)$. The initial amplitude is larger for the noisiest case, which is consistent with a feedback loop. However, the largest amplitude growth is found to correlate with the lowest acoustic emission, as opposed to Rossiter cavity modes.

Figure 17 shows a tendency for the leading 2-D TKE-based POD mode to attenuate from the reattachment point to the trailing edge. This is important, because the acoustic-based POD analysis demonstrated that only 2-D structures are associated with sound. Iso-surfaces of the $\lambda_{2}$ criterion in figure 25 clearly show streamwise oriented vortices in the gap region, similar to the ones identified by Imamura et al. (2008) and Deck \& Laraufie (2013). These vortices are likely to be due to the accelerating 

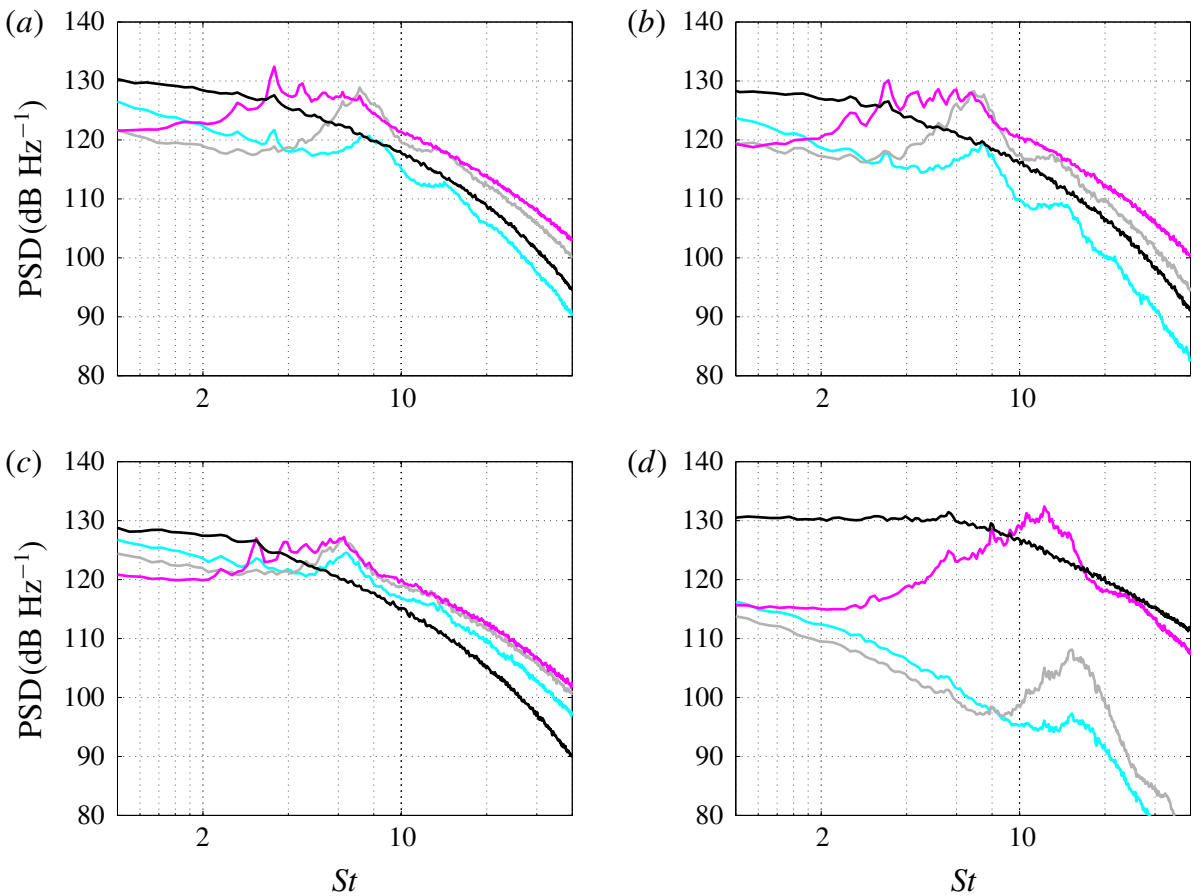

FIGURE 24. (Colour online) PSD of streamwise velocity component in four mixing-layer evolution stages, stage 1 (cyan), stage 2 (grey), stage 3 (magenta), stage 4 (black). Cases (a) olA $\alpha 4,(b)$ olA $\alpha 8,(c)$ olB $\alpha 4$ and $(d)$ olC $\alpha 4$.

flow field in the vicinity of the main element leading edge (see figure 26), as first explained by Batchelor \& Proudman (1954).

Figure 27 shows the evolution of the planar velocity magnitude $\left(\left|\phi_{u v}\right|=\left(\left|\phi_{u}\right|^{2}+\right.\right.$ $\left.\left|\phi_{v}\right|^{2}\right)^{0.5}$, where $\phi_{u}$ and $\phi_{v}$ are the $x$ and $y$ velocity components) of the leading 2-D TKE-based POD mode for representative frequencies. The time-spanwise-averaged flow speed along the disturbance path is also shown in the figure revealing that the energy decay of the 2-D disturbances correlates well with the flow acceleration downstream of the reattachment point. In turn, the magnitude of flow acceleration correlates well with the noise suppression. This observation is consistent with the argument that the accelerating flow field favours three-dimensionalization of the mixing-layer structures, reducing the sound emission. This may explain the variation of sound emission observed for the different configurations and, in particular, the absence of the narrowband peaks in the olC $\alpha 4$ case noise spectrum.

\section{Conclusions}

This paper presents unsteady three-dimensional simulations of the flow over different high-lift configurations based on the MD30P30N three-element airfoil with the aim of understanding the generation mechanism of the low-frequency narrowband peaks. Different tests are done to ensure the robustness of the results with respect to the choice of computational parameters. Additionally, the quality of the simulation data is verified by comparisons with wind tunnel measurements.

Proper orthogonal decomposition using two different metrics is applied with the aim of identifying the flow structures responsible for the noise generation. POD 
(a)

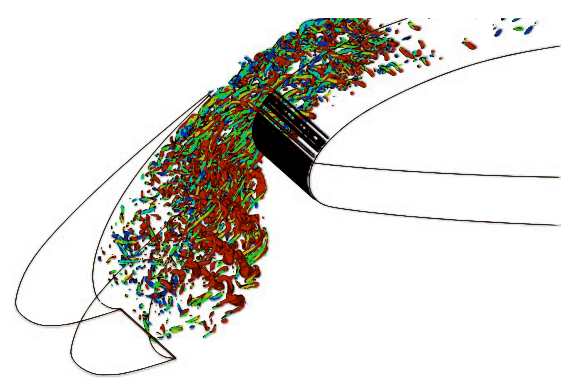

(c)

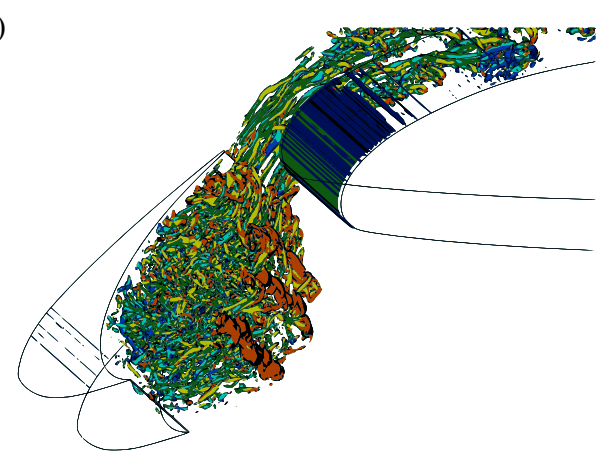

(b)

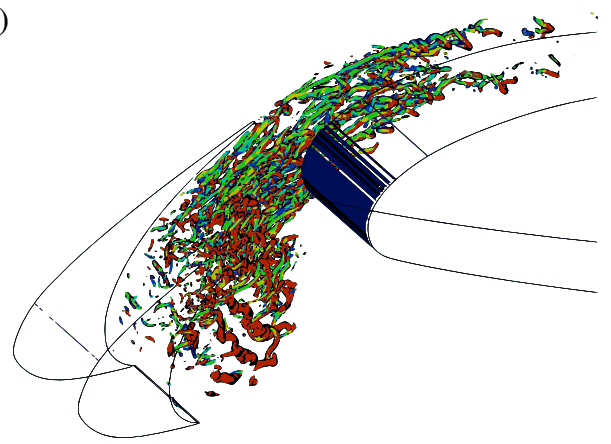

(d)

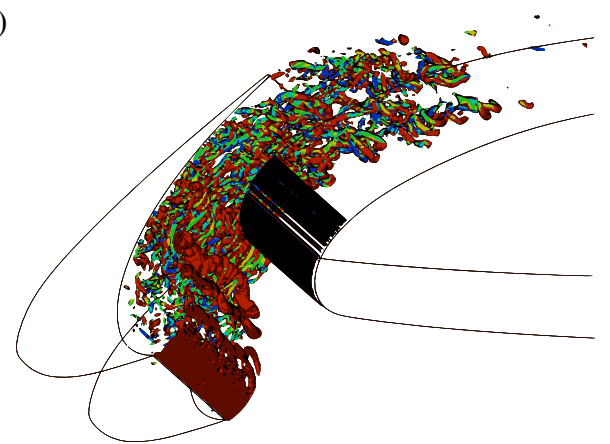

FIgURE 25. (Colour online) Iso-surfaces of $\lambda_{2}$ in the slat cove. (a) $\lambda_{2}=-20000$ for olA $\alpha 4 ;(b) \lambda_{2}=-20000$ for olA $\alpha 8 ;(c) \lambda_{2}=-5000$ for olB $\alpha 4 ;(d) \lambda_{2}=-20000$ for olC $\alpha 4$.

results using the turbulent kinetic energy metric show that, at narrowband peak frequencies, spanwise aligned structures resembling Kelvin-Helmholtz vortices are the most energetic ones, and their characteristics relate them to the mixing-layer primary instability, while irregular structures of indistinct orientation dominate fluctuations in the slat cove at the frequencies of low noise emission. On the other hand, POD results considering the acoustic pressure metric show that the structures responsible for the low-frequency sound are spanwise-coherent $\mathrm{K}-\mathrm{H}$ vortices, for both peaks and broadband noise. The first normalized acoustic-pressure-based POD value reaches levels higher than $95 \%$ in some cases, which contrasts with the rather low values, below $40 \%$ in the best cases, when the turbulent kinetic energy metric is used. This demonstrates that the far-field noise is, for each frequency, mostly associated with a single POD mode captured by the acoustic pressure metric.

The existence of a Rossiter-like feedback mechanism is investigated using acoustic-based POD modes. At frequencies of narrowband peaks, these POD modes are consistent with a feedback cycle where sound waves are emitted as the $\mathrm{K}-\mathrm{H}$ structures interact with the slat trailing edge and these waves introduce disturbances in the mixing layer at the slat cusp. In accordance to what was observed by Rossiter, for the POD modes to close the cycle a phase shift of $\pi / 2$ must be considered between the hydrodynamic and acoustic disturbance at the slat trailing edge.

Finally, the present results suggest that the amplitude of the narrowband peaks is governed by the flow acceleration in the region between the mixing-layer reattachment and the slat trailing edge. Intense flow accelerations in the slat gap region associated with the main element's suction peak appear to be the origin of the streamwise 
(a)

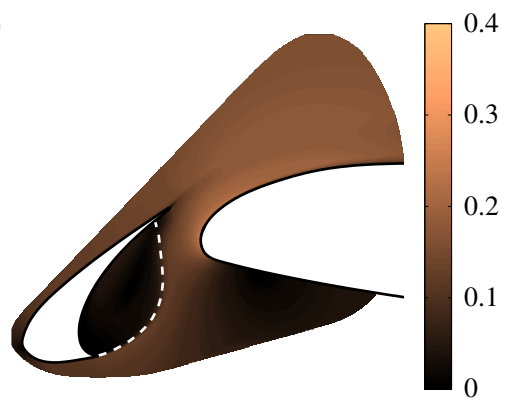

(c)

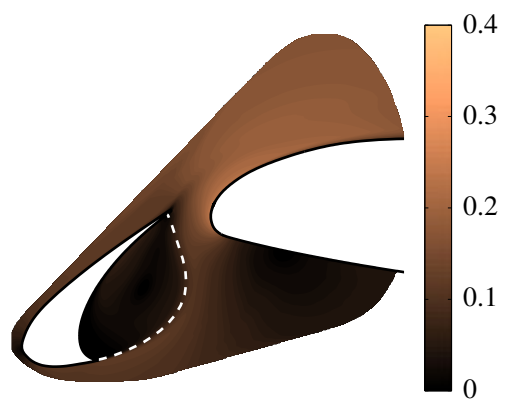

(b)

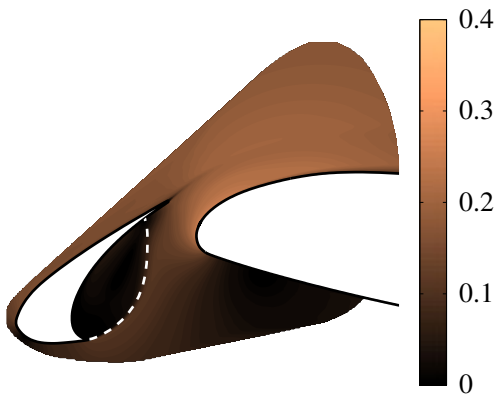

(d)

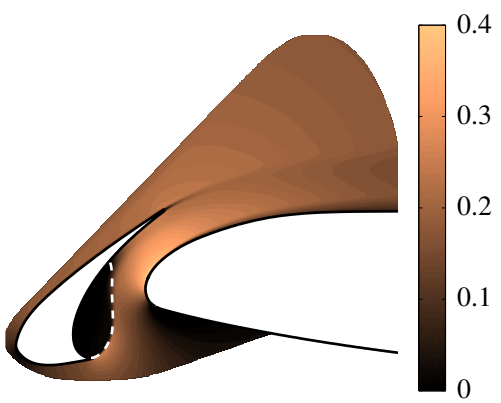

FIgURE 26. (Colour online) Averaged Mach number field in the slat cove region. Cases (a) olA $\alpha 4$; (b) olA $\alpha 8$; (c) olB $\alpha 4 ;$ (d) olC $\alpha 4$.

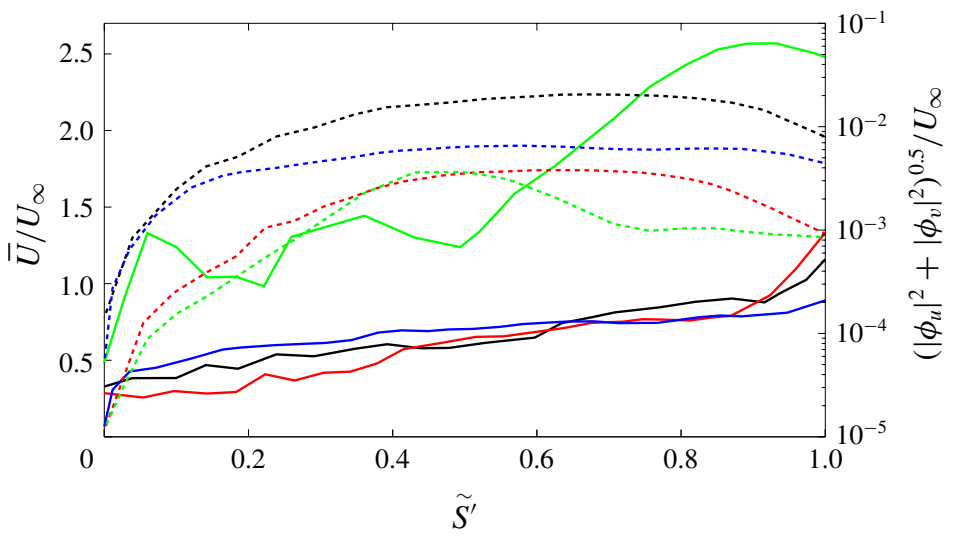

FIGURE 27. (Colour online) Averaged velocity (solid lines) and amplitude of planar velocity components of the turbulent-kinetic-energy-based dominant POD modes. Black: $S t=3.53$ for case $o l A \alpha 4$, red: $S t=3.31$ for case $o l A \alpha 8$, blue: $S t=3.09$ for case $o l B \alpha 4$ and green: $S t=5.67$ for case $o l C \alpha 4$.

vortices observed in the simulations and recovered as POD modes using the turbulent kinetic energy metric. As a consequence, the fluctuations' spanwise coherence in the gap flow is reduced and the trailing-edge sound emission becomes less efficient, explaining the relation between the main-element suction peak and narrowband noise amplitudes observed in experiments. This conclusion was only possible because the 
two different POD metrics used revealed that, even when 3-D structures are dominant, only the 2-D spanwise structures are associated with the sound emission.

The observation that the flow acceleration caused by the main-element suction peak reduces the noise is of fundamental importance, since the slat is designed to reduce the main element's suction peak and, as a consequence, inhibits this mechanism of noise suppression. The difficulty in combining good aerodynamic performance and low noise in the slat is apparent in Pott-Pollenske et al. (2003) and Pagani et al. (2017). Present results offer an explanation for this observation.

\section{Acknowledgements}

D.S.S. received funding from Coordination for the Improvement of Higher Education Personnel (CAPES/Brazil), grant no. DS00011/07-0. D.R. acknowledges funding by $\mathrm{CNPq} /$ Brazil (grant nos. 405144/2016-4, 305512/2016-1) and FAPERJ/ Brazil (grants E-26/010.000356/2017 and E-26/200.003/2018). F.H.T.H. received financial support from São Paulo Research Foundation (FAPESP/Brazil) (grant nos 2016/02970-5, 2018/02542-9). M.A.F.M. is sponsored by CNPq/Brazil (grant nos 304243/2013-2, 304859/2016-8). The initial stages of this research were financed by FAPESP/Brazil (grant no. 2006/52568-7). D.S.S and M.A.F.M acknowledge support from Marie Curie Grant PIRSES-GA-2009-247651 (FP7-PEOPLE-IRSES: ICOMASEF, Instability and COntrol of MAssively SEparated Flows). The authors are also grateful to EMBRAER, EXA Corp and Núcleo de Dinâmica dos Fluidos (NDF/USP) for providing the PowerFLOW licence.

\section{REFERENCES}

Aflalo, B., Simões, L. G. C., Silva, R. \& Medeiros, M. A. F. 2010 Comparative analysis of turbulence models for slat noise source calculations employing unstructured meshes. In Proceedings of the 16th AIAA/CEAS Aeroacoustics Conference. American Institute of Aeronautics and Astronautics.

Amaral, F., Himeno, F., Pagani, C. JR. \& Medeiros, M. 2017 Slat noise from an MD30P30N airfoil at extreme angles of attack. AIAA J. 56 (3), 964-978.

Amaral, F. R., Pagani, C. C. JR., Himeno, F. H .T., Souza, D. D. \& Medeiros, M. A. F. 2019 On closed-section wind-tunnel aeroacoustic experiments with a two-dimensional lifting body. Appl. Acoust. 148, 409-422.

Arndt, R. E. A., Long, D. F. \& Glauser, M. N. 1997 The proper orthogonal decomposition of pressure fluctuations surrounding a turbulent jet. J. Fluid Mech. 340, 1-33.

Batchelor, G. K. \& Proudman, I. 1954 The effect of rapid distortion of a fluid in turbulent motion. Q. J. Mech. Appl. Maths 7 (1), 83-103.

Bhatnagar, P. L., Gross, E. P. \& KrooK, M. 1954 A model for collision processes in gases. I. Small amplitude processes in charged and neutral one-component systems. Phys. Rev. 94 (3), 511-525.

BrÈs, G. A., PÉRot, F. \& Freed, D. 2010 A Ffowcs Williams-Hawkings solver for lattice-Boltzmann based computational aeroacoustics. In Proceedings of the 16th AIAA/CEAS Aeroacoustics Conference. American Institute of Aeronautics and Astronautics.

Chin, V. D., Peters, D. W., Spaid, F. W. \& McGhee, R. J. 1993 Flowfield measurements about a multi-element airfoil at high Reynolds numbers. In Proceedings of the 24th AIAA Fluid Dynamics Conference. American Institute of Aeronautics and Astronautics.

Choudhari, M., Lockard, D., Macaraeg, M., Singer, B., Streett, C., Neubert, G., Stoker, W., Underbrink, J., Berkman, M., Khorrami, M. et al. 2002 Aeroacoustic experiments in the Langley low-turbulence pressure tunnel. Tech. Rep. NASA, Hampton, USA. 
Choudhari, M. M. \& Khorrami, M. R. 2007 Effect of three-dimensional shear-layer structures on slat cove unsteadiness. AIAA J. 45 (9), 2174-2186.

Crighton, D. G. 1991 Airframe noise. In Aeroacoustics of Flight Vehicles: Theory and Practice (ed. H. H. Hubbard), vol. 1, p. 447. NASA.

Dantas, L., Catalano, F., Medeiros, M. \& Carmo, M. 2010 The update process and characterization of the Sáo Paulo wind-tunnel for aeroacoustic testing. In Proceedings ICAS-2010. Optimage Ltd.

DECK, S. 2005 Zonal-detached-eddy simulation of the flow around a high-lift configuration. AIAA J. 43 (11), 2372-2384.

DeCK, S. \& Laraufie, R. 2013 Numerical investigation of the flow dynamics past a three-element aerofoil. J. Fluid Mech. 732, 401-444.

Dierke, J., Appel, C., Siebert, J., Bauer, M., Siefert, M. \& Ewert, R. 2011 3D computation of broadband slat noise from swept and unswept high-lift wing sections. In Proceedings of the 17th AIAA/CEAS Aeroacoustics Conference. American Institute of Aeronautics and Astronautics.

DobRZYNSKI, W. 2010 Almost 40 years of airframe noise research: what did we achieve? J. Aircraft 47 (2), 353-367.

Dobrzynski, W., Nagakura, K., Gehlhar, B. \& Bushbaum, A. 1998 Airframe noise studies on wings deployed high-lift devices. In Proceedings of the 4th AIAA/CEAS Aeroacoustics Conference. American Institute of Aeronautics and Astronautics.

Dobrzynski, W. \& Pott-Pollenske, M. 2001 Slat noise source studies for farfield noise prediction. In Proceedings of the 7th AIAA/CEAS Aeroacoustics Conference. American Institute of Aeronautics and Astronautics.

Druault, P., Gloerfelt, X. \& Mervant, T. 2011 Investigation of flow structures involved in sound generation by two- and three-dimensional cavity flows. Comput. Fluids 48, 54-67.

EWERT, R. 2008 Broadband slat noise prediction based on CAA and stochastic sound sources from a fast random particle-mesh (RPM) method. Comput. Fluids 37, 369-387.

Ewert, R., Dierke, J., Pott-Pollenske, M., Appel, C., Emunds, R. \& Sutcliffe, M. 2010 CAA-RPM prediction and validation of slat setting influence on broadband high-lift noise generation. In Proceedings of the 16th AIAA/CEAS Aeroacoustics Conference. American Institute of Aeronautics and Astronautics.

FARASSAT, F. \& SUCCI, G. 1980 A review of propeller discrete frequency noise prediction technology with enphasis on two current methods for time domain calculations. Comput. Fluids 35, 898-909.

FARES, E. 2006 Unsteady flow simulation of the ahmed reference body using a lattice Boltzmann approach. Computers and Fluid 35, 940-950.

FfowCS-WiLliams, J. \& HALL, L. 1970 Aerodynamic sound generation by turbulence in the vicinity of a scattering half plane. J. Fluid Mech. 40, 657-670.

FINK, M. R. 1979 Noise component method for airframe noise. J. Aircraft 16 (10), 659-665.

Freund, J.\& Colonius, T. 2009 Turbulence and sound-field POD analysis of a turbulent jet. Intl J. Aeroacoust. 8 (4), 337-354.

He, X. \& Luo, L. 1997 Theory of lattice Boltzmann method: from the Boltzmann equation to the lattice Boltzmann equation. Phys. Rev. E 56 (6), 6811-6817.

Hein, S., Hohage, T., Koch, W. \& Schöberl, J. 2007 Acoustic resonances in a high-lift configuration. J. Fluid Mech. 582, 179-202.

Heller, H. \& Bliss, D. 1975 The physical mechanism of flow-induced pressure fluctuations in cavities and concepts for their suppression. In Proceedings of the 2nd AIAA Aeroacoustics Conference. American Institute of Aeronautics and Astronautics.

Herr, M., Pott-Pollenske, M., Ewert, R., Boenke, D., Siebert, J., Delfs, J., Rudenko, A., BÜscher, A., Friedel, H. \& MARIOTTI, I. 2015 Large-scale studies on slat noise reduction. In Proceedings of the 21st AIAA/CEAS Aeroacoustics Conference. American Institute of Aeronautics and Astronautics.

Holmes, P., Lumley, J. \& Berkooz, G. 1996 Turbulence, Coherent Structures, Dynamical Systems and Symmetry. Cambridge University Press. 
Imamura, T., Enomoto, S., Yokokawa, Y. \& Yamamoto, K. 2008 Three-dimensional unsteady flow computations around a conventional slat of high-lift devices. AIAA J. 46 (5), 1045-1053.

Imamura, T., Ura, H., Yokokawa, Y. \& Yамамото, K. 2009 A far-field noise and near-field unsteadyness of a simplified high-lift-configuration model (slat). In Proceedings of the 15th AIAA/CEAS Aeroacoustics Conference. American Institute of Aeronautics and Astronautics.

Jenkins, L. N., Khorrami, M. R. \& Choudhari, M. M. 2004 Characterization of unsteady flow structures near leading-edge slat: part I. PIV measurements. In Proceedings of the 10th AIAA/CEAS Aeroacoustics Conference. American Institute of Aeronautics and Astronautics.

Khorrami, M., Berkman, M. \& Choudhari, M. 2000 Unsteady flow computation of a slat with a blunt trailing edge. AIAA J. 38 (11), 2050-2058.

Khorrami, M. R., Choudhari, M. M. \& Jenkins, L. N. 2004 Characterization of unsteady flow structures near leading-edge slat: part II. 2D computations. In Proceedings of the 10th AIAA/CEAS Aeroacoustics Conference. American Institute of Aeronautics and Astronautics.

Khorrami, M. R., Singer, B. \& Berkman, M. 2001 Time-accurate simulations and acoustic analysis of slat free-shear-layer. In Proceedings of the 7th AIAA/CEAS Aeroacoustics Conference. American Institute of Aeronautics and Astronautics.

Khorrami, M. R., Singer, B. \& Lockard, D. 2002 Time-accurate simulations and acoustic analysis of slat free-shear-layer: part II. In Proceedings of the 8th AIAA/CEAS Aeroacoustics Conference. American Institute of Aeronautics and Astronautics.

Klausmayer, S. M. \& Lin, J. C. 1994 An experimental investigation of skin friction on a multi-element airfoil. In Proceedings of the 12th AIAA Applied Aerodynamics Conference. American Institute of Aeronautics and Astronautics.

Kolb, A., Faulhaber, P., Drobietz, R. \& Grünewald, M. 2007 Aeroacoustic wind tunnel measurements on a 2D high-lift configuration. In Proceedings of the 13th AIAA/CEAS Aeroacoustics Conference. American Institute of Aeronautics and Astronautics.

LI, Y., SHOck, R., ZHANG, R. \& CHEN, H. 2004 Numerical study of flow past an impulsively started cylinder by the lattice-Boltzmann method. J. Fluid Mech. 519, 273-300.

Lockard, D. P. \& Choudhari, M. M. 2009 Noise radiation from leading-edge slat. In Proceedings of the 15th AIAA/CEAS Aeroacoustics Conference. American Institute of Aeronautics and Astronautics.

LOcKard, D. P. \& Choudhari, M. M. 2012 The influence of realistic Reynolds numbers on slat noise simulations. In Proceedings of the 18th AIAA/CEAS Aeroacoustics Conference. American Institute of Aeronautics and Astronautics.

Mendoza, J., Brooks, T. \& Humphreys, W. JR. 2002 An aeroacoustic study of a leading edge slat configuration. Intl J. Aeroacoust. 1 (3), 241-274.

Murayama, M., Nakakita, K., Yamamoto, K., Ura, H., Ito, Y. \& Choudhari, M. 2014 Experimental study of slat noise from 30P30N three-element high-lift airfoil in JAXA hard-wall low-speed wind tunnel. In Proceedings of the 20th AIAA/CEAS Aeroacoustics Conference. American Institute of Aeronautics and Astronautics.

NAJAFI-YAZDI, A., BrÈs, G. \& MongeAU, L. 2011 An acoustic analogy formulation for moving sources in uniformly moving media. Proc. R. Soc. A 467, 144-165.

Pagani, C. JR., SouZA, D. \& Medeiros, M. 2016 Slat noise: aeroacoustic beamforming in closedsection wind tunnel with numerical comparison. AIAA J. 54 (7), 2100-2115.

Pagani, C. JR., Souza, D. \& Medeiros, M. 2017 Experimental investigation on the effect of slat geometrical configurations on aerodynamic noise. J. Sound Vib. 394, 256-279.

PAscioni, K. A. \& CAtTAfesta, L. N. 2018 Unsteady characteristics of a slat-cove flow field. Phys. Rev. Fluids 3, 034607.

PÉrennè, S. \& Roger, M. 1998 Aerodynamic noise of a two-dimensional wing with high-lift devices. In Proceedings of the 4th AIAA/CEAS Aeroacoustic Conference. American Institute of Aeronautics and Astronautics.

Pott-Pollenske, M., Alvarez-Gonzalez, J. \& Dobrzynski, W. 2003 Effect of slat gap/overlap on farfield radiated noise. In Proceedings of 9th AIAA/CEAS Aeracoustics Conference. American Institute of Aeronautics and Astronautics. 
Richard, P. R., Wilkins, S. J. \& Hall, J. W. 2018 Particle image velocity investigation of the coherent structures in a leading-edge slat flow. J. Fluids Engng 582, 179-202.

Rossiter, J. E. 1966 Wind-tunnel experiments on the flow over rectangular cavities at subsonic and transonic speeds. Tech. Rep. Aeronautical Research Council.

Rowley, C. W., Colonius, T. \& BAsu, A. 2002 On self-sustained oscilations in two-dimensional compressible flow over rectangular cavity. J. Fluid Mech. 455, 315-346.

SAtTi, R., Li, Y., Shock, R. \& Noelting, S. 2008 Simulation of flow over a 3-element airfoil using a lattice-Boltzmann method. In Proceedings of the 46th AIAA Aerospace Sciences Meeting and Exhibit. American Institute of Aeronautics and Astronautics.

Simões, L. G. C., SouzA, D. S. \& Medeiros, M. A. F. 2011 On the small effect of boundary layer thicknesses on slat noise. In Proceedings of the 17th AIAA/CEAS Aeroacoustics Conference. American Institute of Aeronautics and Astronautics.

Sinha, A., Rodríguez, D., Brès, G. \& Colonius, T. 2014 Wavepacket model for supersonic jet noise. J. Fluid Mech. 742, 71-95.

SIROVICH, L. 1987 Turbulence and the dynamics of coherent structures. Parts I-III. Q. Appl. Maths 45 (3), 561-571.

Souza, D. S., Rodríguez, D., Simões, L. \& Medeiros, M. A. F. 2015 Effect of an excrescence in the slat cove: flow-field, acoustic radiation and coherent structures. Aerosp. Sci. Technol. 44, 108-115.

SPAID, F. W. \& LYNCH, F. T. 1996 High Reynolds number, multi-element airfoil flowfield measurements. In Proceedings of the 34th AIAA Aerospace Sciences Meeting and Exhibit. American Institute of Aeronautics and Astronautics.

Sun, Y., Taira, K., Cattafesta III, L. N. \& Ukeiley, L. S. 2017 Biglobal instabilities of compressible open-cavity flows. J. Fluid Mech. 826, 270-301.

TeiXeIRA, C. M. 1998 Incorporating turbulence models into the Lattice-Boltzmann method. Intl J. Mod. Phys. C 9 (8), 1159-1175.

Terracol, M., Manoha, E. \& Lemoine, B. 2016 Investigation of the unsteady flow and noise generation in a slat cove. AIAA J. 54 (2), 469-489.

Valarezo, W. O., Dominik, C. J., McGhee, R. J., Goodman, W. L. \& Paschal, K. B. 1991 Multielement airfoil optimization for maiximum lift at high Reynolds numbers. In Proceedings of 9th AIAA Applied Aerodynamics Conference. American Institute of Aeronautics and Astronautics.

Wolf-Gladrow, D. 2000 Lattice-Gas Cellular Automata and Lattice Boltzmann Models: An Introduction. Springer.

ZHANG, Y., Chen, H., WANG, K. \& WANG, M. 2017 Aeroacoustic prediction of a multi-element airfoil using wall-modeled large-eddy simulation. AIAA J. 55 (12), 4219-4233. 